\title{
Structure of Korteweg models and stability of diffuse interfaces
}

\author{
S. BENZONI-GAVAGE ${ }^{\dagger}$ \\ Institut Camille Jordan, Université Claude Bernard Lyon I, 43, boulevard du 11 novembre 1918, \\ F-69622 Villeurbanne Cedex, France \\ R. DANCHIN ${ }^{\ddagger}$ \\ Centre de Mathématiques - Faculté de Sciences et Technologie, Université Paris XII, \\ 61, avenue du Général de Gaulle, F-94010 Créteil Cedex, France \\ S. DESCOMBES $\$$ \\ UMPA - ENS Lyon, 46, allée d'Italie, F-69364 Lyon Cedex 07, France \\ AND \\ D. JAMET ${ }^{\mathrm{II}}$ \\ DER / SSTH / LMDL, CEA-Grenoble, 17 rue des martyrs, F-38054 Grenoble Cedex 9, France
}

[Received 11 November 2004 and in revised form 6 June 2005]

The models considered are supposed to govern the motion of compressible fluids such as liquidvapor mixtures endowed with a variable internal capillarity. Several formulations and simplifications are discussed, from the full multi-dimensional equations for non-isothermal motions in Eulerian coordinates to the one-dimensional equations for isothermal motions in Lagrangian coordinates. Hamiltonian structures are pointed out in each case, and in the one-dimensional isothermal case, they are used to study the stability of two kinds of non-linear waves: the solitary, or homoclinic waves, and the heteroclinic waves, which correspond to propagating phase boundaries of non-zero thickness, also called diffuse interfaces. It is known from an earlier work by Benzoni-Gavage [Phys. D, 2001] that the latter are (weakly) spectrally stable. Here, diffuse interfaces are shown to be orbitally stable. The proof relies on their interpretation as critical points of the Hamiltonian under constraints, whose justification requires some care because of the different endstates at infinity. Another difficulty comes from higher order derivatives that are not controlled by the Hamiltonian. In the case of a variable capillarity, our stability result unfortunately does not imply global existence. As regards the solitary waves, which come in families parametrized by the wave speed, they are not stable from the variational point of view. However, using a method due to Grillakis, Shatah and Strauss [J. Funct. Anal., 1987], it is possible to show that some solitary waves, depending on their speed, are orbitally stable. Namely, the convexity of a function of the wave speed called moment of instability determines the stability of solitary waves. This approach, already used by Bona and Sachs [Comm. Math. Phys., 1988] for the Boussinesq equation, is here adapted to solitary waves in Korteweg models, which are first classified according to their endstate and internal structures. The corresponding moments of instability are computed by quadrature. They exhibit both convexity and concavity regions.

2000 Mathematics Subject Classification: 76T10, 35B35, 35Q51, 37C29.

\footnotetext{
†E-mail: benzoni@maply.univ-lyon1.fr

‡E-mail: danchin@univ-paris12.fr

${ }^{\S}$ E-mail: sdescomb@umpa.ens-lyon.fr

${ }^{\text {II }}$ E-mail: didier.jamet@cea.fr
} 
Keywords: Hamiltonian structure; conservation laws; capillarity; diffuse interface; soliton; orbital stability.

\section{Introduction}

The aim of this paper is to investigate the asymptotic behavior of Korteweg models for compressible fluids endowed with internal capillarity and possibly exhibiting phase changes. The models we consider are originated from the XIXth century work by van der Waals [33] and Korteweg [26] and were actually derived in their modern form in the 1980s using the second gradient theory (see for instance [36, 23]). We point out that special cases of these models have also arisen in other contexts, e.g. in the water waves theory and more recently in quantum hydrodynamics. Our main motivation comes from compressible fluids though, especially liquid-vapor mixtures with phase changes, and we are interested in the propagation of two kinds of non-linear waves in those media: the solitary waves, which are perturbations with a limited expanse propagating in a single stable phase, and the propagating boundaries between two stable phases.

A feature of Korteweg models is that they allow "phase boundaries" of non-zero thickness, often called diffuse interfaces by contrast with sharp interfaces in the Laplace-Young theory. The actual width of a liquid-vapor interface is extremely small far away from the critical point, but becomes infinite when the critical temperature is approached from below. (Recall that the "phases" of a fluid are indistinguishable at temperatures larger than a critical temperature, whose value varies from one fluid to another.) The relevance of diffuse interfaces is thus clear near critical point. Furthermore, Korteweg's theory of capillarity provides an intrinsic way-differently from LaplaceYoung's theory - of incorporating surface tension in the models. The interest for diffuse interfaces has been renewed in the late 1990s for numerical reasons. Indeed, across the diffuse interfaces, all the physical variables encounter possibly strong but nevertheless continuous variations. This property allows one to solve the same system of continuous equations everywhere, this system governing the motion of all the "interfaces" regardless of their topology. This feature avoids the numerical difficulties associated to the resolution of moving boundaries problems associated to multi-phase systems (see [2] for a nice review). However, the development of efficient and accurate numerical schemes requires some mathematical knowledge of the system of PDEs to be solved. In particular, stability issues are of special interest.

The mathematical analysis of Korteweg models is rather recent. One may quote only a few papers [12, 15, 19], in which non-zero viscosity and its regularizing effects play a fundamental role. Even more recently, advances have been made in the context of quantum hydrodynamics, in which the equations involve some sort of dissipation-namely, relaxation-and a special, though nonconstant capillarity coefficient [28, 22]. In a companion paper [7] we have addressed the analysis of purely capillary models with variable capillarities. Here we concentrate more on algebraic aspects of these models, and in particular on their Hamiltonian structure. Using Olver's method [30], we show that the natural Hamiltonian formulations - those associated with total energy — are compatible with Jacobi's identity only in dimension one (see Section 1). Further investigations would consist in looking for additional structures. These are known to exist for the Boussinesq equation, which may be viewed as a special case of the models we consider, but they are far less natural than the one associated with the total energy.

Hamiltonian Korteweg models admit several kinds of traveling wave solutions, mainly homoclinic (or solitary) waves, heteroclinic waves, and periodic waves. This is easily shown by 
means of a phase portrait analysis of the governing ODEs, which are themselves Hamiltonian. We concentrate here on homoclinic waves, also called solitons, and heteroclinic waves, which we call kinks. We propose a classification of solitons according to various parameters, depending on the bulk phase and on whether the fluid is compressed or expanded inside the "bump". Propagating phase boundaries belong to kinks, but there are also kinks which cannot be termed phase boundaries, as they occur in fluids above the critical temperature. Both kinds of waves, solitons and kinks, are characterized by a persistent shape, or profile, traveling at constant speed and which is flat at infinity because the endstates are (generically) hyperbolic fixed points of the governing ODEs. There is however a very important qualitative difference between those waves. Indeed, for fixed endstates, solitons come in one-parameter families (parametrized by the mass transfer flux across the wave, or equivalently by the speed of the wave) whereas kinks are uniquely determined by either one of the endstates. From a spectral stability point of view, the second situation is better, as we show in Section 3, and therefore the non-linear stability analysis is to some extent simpler. For solitons, a more sophisticated tool is needed to circumvent the difficulty associated with an unstable eigenvalue. Such a tool was introduced in an abstract setting by Grillakis, Shatah and Strauss [18], and applied in [9] to the water waves governed by the Boussinesq equation. A slight generalization makes it available in our context. After Boussinesq and Bona-Sachs [9], we call that tool the moment of instability of a solitary wave. It defines a smooth function on the manifold of solitons, and in particular along the curves made of solitons having the same endstates. Its local convexity along such a curve determines the stability of a soliton.

In a prior work [5], Benzoni-Gavage investigated the spectral stability of heteroclinic profiles in several space dimensions. The main result is that the spectrum of the spatial operator in the linearized equations about a given heteroclinic profile coincides with the purely imaginary axis. This is a rather strong result, which relies on tricky energy estimates. However, the route to non-linear stability is far from being obvious. In particular, the techniques developed recently by Zumbrun $e t$ al. for other kinds of heteroclinic traveling waves, in particular for viscous shocks [40, 39, 29, 21] or diffusive-dispersive shocks [20], do not apply. Indeed, the presence of essential spectrum on the whole imaginary axis precludes contour shiftings and steepest descent techniques (although these techniques are likely to work for dissipative diffuse interfaces, for which we unfortunately lack information on spectral stability).

In one space dimension however, the Hamiltonian framework helps. Indeed, traveling profiles may be viewed as critical points of the Hamiltonian under constraints, these constraints being linked to additional conservation laws. We use this crucial observation here to define the moment of instability $m$ of any soliton. Furthermore, we point out that $m$ can be evaluated in the phase plane, without knowing explicitly the profile of the soliton. (In fact, $m$ appears to coincide with what is usually recognized as surface tension in equilibrium phase boundaries!) In other words, $m$ is given by a definite integral, which can be evaluated numerically with high precision. Then it is easy to check numerically, or even graphically, whether the graph of $m$ over solitons with the same endstates is convex. We provide plots of $m$ for the various classes of solitons we have identified. Those which propagate in stable phases, regardless of the stability of the states inside the bump, appear to be stable. Of course by stable we mean only orbitally stable, because of the translational invariance. Moreover, in the most general, quasilinear case the stability is limited in time, because the Hamiltonian does not control the "high norm" in which we have local-in-time existence [7] (our stability result is to some extent similar to one known for peakons [14] in the Camassa-Holm equation). When the capillarity coefficient is such that the PDEs are semi-linear, this difficulty can be overcome by means of higher order energy estimates [9], and in this case the stability is global 
in time. Even in this case though, the stability is weaker than in the presence of dissipation: there is no control of the asymptotic shift, nor a decay rate.

As regards heteroclinic profiles, the stability analysis does not require the function $m$ (even though the surface tension to which it corresponds is certainly physically relevant). The important point is that, unlike what happens for homoclinic profiles, the components of heteroclinic profiles are monotone. This enables us to prove that the Hessian of the constrained Hamiltonian does not have any negative eigenvalue. This is what we meant above by "better spectral stability". On the other hand, we have to deal with the different endstates. This requires some care in the definition of the various functionals (the Hamiltonian and the constraints). Furthermore, the fact that the manifold made of all translated profiles is unbounded in Sobolev spaces (or even in $L^{2}$ ) is another obstacle to global stability.

In several space dimensions, the non-linear stability of traveling profiles remains an open problem, since no variational interpretation of profiles is available. As regards local-in-time behavior, we refer to [6].

\section{Hamiltonian structures for Korteweg models}

\subsection{A general model in Eulerian coordinates}

Korteweg-type models are based on a non-local version of thermodynamics, which assumes that the energy of the fluid not only depends on standard variables but on the gradient of the density. In terms of the free energy for instance, this principle takes the form of a generalized Gibbs relation

$$
\mathrm{d} F=-S \mathrm{~d} T+g \mathrm{~d} \rho+\phi^{*} \cdot \mathrm{d} \mathbf{w}
$$

where $F$ denotes the free energy per unit volume, $S$ the entropy per unit volume $1 T$ the temperature, $g$ the chemical potential and, in the additional term, $\mathbf{w}$ stands for $\nabla \rho$. The potential $\phi$ is most often assumed of the form

$$
\phi=K \mathbf{w}
$$

where $K$ is called the capillarity coefficient, which may depend on both $\rho$ and $T$. In this case, $F$ decomposes into a standard part $F_{0}$ and an additional term due to gradients of density,

$$
F(\rho, T, \nabla \rho)=F_{0}(\rho, T)+\frac{1}{2} K(\rho, T)\|\nabla \rho\|^{2},
$$

and similar decompositions hold for $S$ and $g$. We shall use this special form in our subsequent analysis. For the moment we keep the abstract potential $\phi$ and we define the Korteweg tensor as

$$
\mathbf{K}:=(\rho \operatorname{div} \phi) \mathbf{I}-\phi \mathbf{w}^{*} .
$$

Neglecting dissipation phenomena 2 the conservation of mass, momentum and energy read

$$
\left\{\begin{array}{l}
\partial_{t} \rho+\operatorname{div}(\rho \mathbf{u})=0, \\
\partial_{t}\left(\rho \mathbf{u}^{*}\right)+\operatorname{div}\left(\rho \mathbf{u} \mathbf{u}^{*}+p \mathbf{I}\right)=\operatorname{div} \mathbf{K}, \\
\partial_{t}\left(E+\frac{1}{2} \rho\|\mathbf{u}\|^{2}\right)+\operatorname{div}\left(\left(E+\frac{1}{2} \rho\|\mathbf{u}\|^{2}+p\right) \mathbf{u}\right)=\operatorname{div}(\mathbf{K u}+\mathbf{W}),
\end{array}\right.
$$

1 By convention, extensive quantities per unit volume are denoted by upper case letters, and their specific counterparts will be denoted by the same lower case letters.

2 As regards heat conductivity, we are aware that this assumption is questionable. 
where $p=\rho g-F$ is the (extended) pressure, $E=F+T S$ is the internal energy per unit volume, and

$$
\mathbf{W}:=\left(\partial_{t} \rho+\mathbf{u}^{*} \cdot \nabla \rho\right) \phi=-(\rho \operatorname{div} \mathbf{u}) \phi
$$

is the interstitial working that was first introduced by Dunn and Serrin [16]. This supplementary term ensures that the entropy $S$ satisfies the conservation law

$$
\partial_{t} S+\operatorname{div}(S \mathbf{u})=0
$$

(This is obtained through formal computation, for presumably smooth solutions.) There is also an alternative form of the momentum equation (still for smooth solutions). Using the mass conservation law and the relation

$$
\mathrm{d} g=-s \mathrm{~d} T+v \mathrm{~d} p+v \phi^{*} \cdot \mathrm{d} \mathbf{w},
$$

with $s$ the specific entropy and $v$ the specific volume, we arrive at the convective equation

$$
\partial_{t} \mathbf{u}+\left(\mathbf{u}^{*} \cdot \nabla\right) \mathbf{u}=\nabla(\operatorname{div} \phi-g)-s \nabla T .
$$

The resulting evolution system for $(\rho, \mathbf{u}, S)$ is

$$
\left\{\begin{array}{l}
\partial_{t} \rho+\operatorname{div}(\rho \mathbf{u})=0 \\
\partial_{t} \mathbf{u}+\left(\mathbf{u}^{*} \cdot \nabla\right) \mathbf{u}=\nabla(\operatorname{div} \phi-g)-s \nabla T \\
\partial_{t} S+\operatorname{div}(S \mathbf{u})=0
\end{array}\right.
$$

Let us consider the total energy $H:=E+\frac{1}{2} \rho\|\mathbf{u}\|^{2}$ as a Hamiltonian. Using the relation

$$
\mathrm{d} E=T \mathrm{~d} S+g \mathrm{~d} \rho+\phi^{*} \cdot \mathrm{d} \mathbf{w},
$$

the variational gradient of the (formal) Hamiltonian functional

$$
\mathcal{H}:=\int H \mathrm{~d} x
$$

is easily found to be

$$
\delta \mathcal{H}=\left(\begin{array}{c}
\frac{1}{2}\|\mathbf{u}\|^{2}+g-\operatorname{div} \phi \\
\rho \mathbf{u} \\
T
\end{array}\right) .
$$

Therefore, the system (1.1) equivalently reads, for irrotational velocity fields,

$$
\partial_{t}\left(\begin{array}{l}
\rho \\
\mathbf{u} \\
S
\end{array}\right)=\mathcal{J} \delta \mathcal{H},
$$

where $\mathcal{J}$ is the skewsymmetric differential operator

$$
\mathcal{J}=\left(\begin{array}{ccc}
0 & -\operatorname{div} & 0 \\
-\nabla & 0 & -s \nabla \\
0 & -\operatorname{div} s . & 0
\end{array}\right)
$$


For the system to fall into the framework of infinite-dimensional Hamiltonian systems, the skewsymmetry of the operator $\mathcal{J}$, which has variable coefficients, is not sufficient. The additional condition is that $\mathcal{J}$ be a Hamiltonian operator, in the sense that the bracket defined for functionals $\mathcal{P}$ and $\mathcal{Q}$ by

$$
\{\mathcal{P}, \mathcal{Q}\}=\int \delta \mathcal{P} \cdot \mathcal{J} \delta \mathcal{Q} \mathrm{d} x
$$

be a genuine Poisson bracket, satisfying the Jacobi identity

$$
\{\{\mathcal{P}, \mathcal{Q}\}, \mathcal{R}\}+\{\{\mathcal{Q}, \mathcal{R}\}, \mathcal{P}\}+\{\{\mathcal{R}, \mathcal{P}\}, \mathcal{Q}\}=0 .
$$

The direct verification of this identity is really tricky in general. Olver's method [30] is a powerful alternative way to do it. The required material and actual computations are given in Appendix A.

PROPOSITION 1 The system (1.1) restricted to irrotational vector fields :

$$
\partial_{t}\left(\begin{array}{l}
\rho \\
\mathbf{u} \\
S
\end{array}\right)=\left(\begin{array}{ccc}
0 & -\operatorname{div} & 0 \\
-\nabla & 0 & -s \nabla \\
0 & -\operatorname{div} s . & 0
\end{array}\right)\left(\begin{array}{c}
\frac{1}{2}\|\mathbf{u}\|^{2}+g-\operatorname{div} \phi \\
\rho \mathbf{u} \\
T
\end{array}\right)
$$

is not Hamiltonian, except in dimension 1.

Another interesting feature of the model (1.1) that was pointed out by Gavrilyuk and Gouin [17] is its symmetrizability when $H$ is convex. More details on the symmetrization may also be found in [7].

We shall now concentrate on the reduced, isothermal model

$$
\left\{\begin{array}{l}
\partial_{t} \rho+\operatorname{div}(\rho \mathbf{u})=0, \\
\partial_{t} \mathbf{u}+\left(\mathbf{u}^{*} \cdot \nabla\right) \mathbf{u}=\nabla(\operatorname{div} \phi-g) .
\end{array}\right.
$$

The isothermal assumption, widely used in physics, facilitates the mathematical analysis of the model. In particular, it yields a simplified Hamiltonian formulation, in which the skewsymmetric operator defining the Poisson bracket has constant coefficients. Indeed, for irrotational velocity fields (1.2) coincides with

$$
\partial_{t}\left(\begin{array}{l}
\rho \\
\mathbf{u}
\end{array}\right)=\mathcal{I} \delta \mathcal{K}, \quad \mathcal{K}=\int\left(F+\frac{1}{2} \rho\|\mathbf{u}\|^{2}\right) \mathrm{d} x, \quad \mathcal{I}=\left(\begin{array}{cc}
0 & -\operatorname{div} \\
-\nabla & 0
\end{array}\right) .
$$

\subsection{Eulerian capillary models}

From now on, we assume that

$$
\phi=K \mathbf{w}
$$

Then we can write

$$
g=g_{0}+\frac{1}{2} K_{\rho}^{\prime}\|\nabla \rho\|^{2},
$$

where $g_{0}$ is independent of $\nabla \rho$. In particular, the isothermal model reduces to

$$
\left\{\begin{array}{l}
\partial_{t} \rho+\operatorname{div}(\rho \mathbf{u})=0 \\
\partial_{t} \mathbf{u}+\left(\mathbf{u}^{*} \cdot \nabla\right) \mathbf{u}=\nabla\left(K \Delta \rho+\frac{1}{2} K_{\rho}^{\prime}\|\nabla \rho\|^{2}-g_{0}\right),
\end{array}\right.
$$


where $g_{0}$ and $K$ are given, smooth functions of $\rho$ (with $K>0$ ). One may also write this system in conservative form, noting that

$$
p=p_{0}+\frac{1}{2}\left(\rho K_{\rho}^{\prime}-K\right)\|\nabla \rho\|^{2}, \quad p_{0}=\rho g_{0}-F_{0},
$$

hence the (complicated) momentum equation

$$
\partial_{t}\left(\rho \mathbf{u}^{*}\right)+\operatorname{div}\left(\rho \mathbf{u u}^{*}\right)+\nabla p_{0}=\nabla\left(\rho K \Delta \rho+\frac{1}{2}\left(K+\rho K_{\rho}^{\prime}\right)\|\nabla \rho\|^{2}\right)-\operatorname{div}(K \nabla \rho \otimes \nabla \rho) .
$$

In one space dimension, the system (1.3) reduces to

$$
\left\{\begin{array}{l}
\partial_{t} \rho+\partial_{x}(\rho u)=0 \\
\partial_{t} u+u \partial_{x} u=\partial_{x}\left(K \partial_{x x}^{2} \rho+\frac{1}{2} K_{\rho}^{\prime}\left(\partial_{x} \rho\right)^{2}-g_{0}(\rho)\right) .
\end{array}\right.
$$

It is interesting to note that models of this kind actually arise in various other contexts. In the special case

$$
K(\rho)=\frac{1}{4 \rho},
$$

the system 1.3 is equivalent-for irrotational flows—-to a non-linear Schrödinger equation known as the Gross-Pitaevskii equation

$$
i \partial_{t} \psi+\frac{1}{2} \Delta \psi=g_{0}\left(|\psi|^{2}\right) \psi
$$

for $\psi=\sqrt{\rho} \mathrm{e}^{i \varphi}, \nabla \varphi=\mathbf{u}$. See for instance [8], where $g_{0}(\rho)=\frac{1}{4} \rho^{2}$. In [28, 22], $K$ is also proportional to $1 / \rho$, and there is almost no restriction on $g_{0}$. One may also observe that, in one dimension with

$$
g_{0}(\rho)=\frac{1}{4} \rho, \quad K(\rho)=\frac{1}{4 \rho},
$$

the equations in (1.4) appear as an equivalent form of the so-called filament equation (see [3, p. 353]).

\subsection{Lagrangian capillary models}

The one-dimensional isothermal model becomes even simpler in Lagrangian formulation. Introducing $y$ the mass Lagrangian coordinate so tha $11 \mathrm{~d} y=\rho \mathrm{d} x-\rho u \mathrm{~d} t$ we obtain with a little piece of calculus the —at least formally—equivalent system

$$
\left\{\begin{array}{l}
\partial_{t} v-\partial_{y} u=0, \\
\partial_{t} u+\partial_{y} p_{0}=-\partial_{y}\left(\kappa \partial_{y y}^{2} v+\frac{1}{2} \kappa_{v}^{\prime}\left(\partial_{y} v\right)^{2}\right),
\end{array}\right.
$$

with

$$
\kappa(v):=K(1 / v)(1 / v)^{5} .
$$

In the special case $\kappa=$ constant, i.e. $K=$ const $\cdot \rho^{-5}$, the system (1.5) is formally equivalent to the (good) Boussinesq equation and is amenable to the theory of Kato [25] (see [9] for more details).

1 This change of variable may be justified rigorously for, say, $C^{1}$ functions $(\rho, u)$ and $\rho$ bounded away from zero. 
Our aim here is to deal with general capillarities, motivated by physical reasons-since there is no reason for $K$ to be proportional to $\rho^{5}$-as well as by the various analogies mentioned above.

Following an idea of F. Coquel [13], we rewrite the velocity equation as

$$
\partial_{t} u+\partial_{y} p_{0}+\partial_{y}\left(\alpha \partial_{y} w\right)=0, \quad \alpha=\sqrt{\kappa}, \quad w=\alpha \partial_{y} v,
$$

and we easily find that $w$ satisfies the equation

$$
\partial_{t} w-\partial_{y}\left(\alpha \partial_{y} u\right)=0 .
$$

Considering $w$ as an additional unknown, we are led to the system

$$
\left\{\begin{array}{l}
\partial_{t} v-\partial_{y} u=0, \\
\partial_{t} u+\partial_{y} p_{0}+\partial_{y}\left(\alpha \partial_{y} w\right)=0, \\
\partial_{t} w-\partial_{y}\left(\alpha \partial_{y} u\right)=0 .
\end{array}\right.
$$

One may observe that 1.6 is again associated with a Hamiltonian formulation. Indeed, redefining $H$ as

$$
H(v, u, w)=f_{0}(v)+\frac{1}{2} u^{2}+\frac{1}{2} w^{2}, \quad f_{0}:=v F_{0},
$$

and $\mathcal{H}=\int H \mathrm{~d} x$, we have

$$
\delta \mathcal{H}=\left(\begin{array}{c}
-p_{0}(v) \\
u \\
w
\end{array}\right)
$$

and 1.6 coincides with

$$
\partial_{t}\left(\begin{array}{c}
v \\
u \\
w
\end{array}\right)=\mathcal{A}(v) \delta \mathcal{H}, \quad \mathcal{A}(v)=\left(\begin{array}{ccc}
0 & \partial_{y} & 0 \\
\partial_{y} & 0 & -\partial_{y} \alpha(v) \partial_{y} \\
0 & \partial_{y} \alpha(v) \partial_{y} & 0
\end{array}\right) .
$$

The operator $\mathcal{A}(v)$ is obviously skewsymmetric. Using again Olver's method [30], we can check that $\mathcal{A}(v)$ is indeed a Hamiltonian operator. This is due to the fact that the coefficient $\alpha$ appearing on the second and third row does not depend on the second and third variables, $u$ and $w$. See Appendix A for the details.

\section{Traveling wave solutions of Korteweg models}

A traveling wave is by definition a solution that propagates the same profile at constant speed. We are interested here in traveling waves achieving constants at infinity, which means that their profile describes a connecting orbit of a certain system of ordinary differential equations. When the connecting orbit is homoclinic, that is, when the endstates are equal, the profile is usually called a soliton. When the connecting orbit is heteroclinic, i.e. with different endstates, the profile is sometimes called a kink and it is the term we shall use.

Both systems (1.4) and (1.5) do admit traveling wave solutions of both kinds, solitons and kinks. This is related to the fact, proved in a general setting in [4, pp. 11-12], that the ODE systems governing connecting orbits are themselves Hamiltonian. In fact, they reduce to planar Hamiltonian systems, for which the phase portrait analysis is quite elementary. 
Let us consider first (1.4). A traveling wave of speed $\sigma$ is a special solution of the form $(\rho, u)=$ $(\rho(x-\sigma t), u(x-\sigma t))$ with

$$
\left\{\begin{array}{l}
(\rho(u-\sigma))^{\prime}=0, \\
(u-\sigma) u^{\prime}=\left(K(\rho) \rho^{\prime \prime}+\frac{1}{2} K(\rho)^{\prime} \rho^{\prime}-g_{0}(\rho)\right)^{\prime},
\end{array}\right.
$$

where ' denotes the derivative with respect to $\xi:=x-\sigma t$. Alternatively, the second equation may be rewritten as

$$
\left(\rho(u-\sigma) u+\widetilde{p}_{0}(\rho)\right)^{\prime}=\left(\rho K(\rho) \rho^{\prime \prime}-\frac{1}{2} K(\rho)\left(\rho^{\prime}\right)^{2}+\frac{1}{2} \rho \rho^{\prime} K(\rho)^{\prime}\right)^{\prime} .
$$

We have denoted here $\widetilde{p}_{0}(\rho)=p_{0}(1 / \rho)$ to avoid confusion. We shall sometimes omit the tilde. (Equation 2.8 may be obtained directly from the conservative form of the momentum equation, or from a slight manipulation of (2.7).) The first equation in 2.77 implies that $\rho(u-\sigma) \equiv$ const. This constant must of course be equal to $j:=\rho_{ \pm}\left(u_{ \pm}-\sigma\right)$ if

$$
\lim _{x \rightarrow \pm \infty}(\rho, u)(x)=\left(\rho_{ \pm}, u_{ \pm}\right) .
$$

In the case of a kink, the equality $\rho_{-}\left(u_{-}-\sigma\right)=\rho_{+}\left(u_{+}-\sigma\right)$ is consistent with the RankineHugoniot condition for the corresponding discontinuous traveling wave. In any case, the quantity $j$ corresponds to the mass flux of fluid across the moving boundary $x=\sigma t$. Additionally, (2.8) shows that for a kink attaining its limits $\rho_{ \pm}$fast enough so that $\rho^{\prime}$ and $\rho^{\prime \prime}$ tend to 0 at infinity, we must have

$$
j u_{-}+p_{0}\left(v_{-}\right)=j u_{+}+p_{0}\left(v_{+}\right),
$$

which is also part of the classical Rankine-Hugoniot condition. In particular for $j=0$, that is, with no mass flux across the moving boundary, the pressures on both sides must be equal. This is a well known condition for the so-called static or equilibrium interfaces. In general, the elimination of the speeds $u_{ \pm}$yields

$$
j^{2}=\frac{p_{0}\left(v_{+}\right)-p_{0}\left(v_{-}\right)}{v_{-}-v_{+}},
$$

which means that the slope of the chord between the endstates in the volume-pressure plane must be negative. Physically, if the kink is a liquid-vapor interface, this means that the pressure must be higher in the liquid, whatever the nature of the phase change (evaporation or condensation). Let us now look at the second equation in 2.7 . It implies

$$
-K(\rho) \rho^{\prime \prime}-\frac{1}{2} K(\rho)^{\prime} \rho^{\prime}+g_{0}(\rho)+\frac{1}{2} u^{2}-\sigma u \equiv \mathrm{constant}=: q,
$$

and if $\rho^{\prime}, \rho^{\prime \prime}$ do vanish at infinity, the constant $q$ must be equal to

$$
g_{0}\left(\rho_{-}\right)+\frac{1}{2} u_{-}^{2}-\sigma u_{-}=g_{0}\left(\rho_{+}\right)+\frac{1}{2} u_{+}^{2}-\sigma u_{+} .
$$

In the case of a kink, this equality is not a consequence of the Rankine-Hugoniot condition. Rather, it is an additional relation, sometimes called the kinetic relation [1, 27]. One may observe that 2.10) reduces to $g_{0}\left(\rho_{-}\right)=g_{0}\left(\rho_{+}\right)$when $j=0$. The equality of chemical potentials on both sides of an equilibrium liquid-vapor interface is well known (see for instance [34, p. 42]). Equation (2.10) is just a dynamical version of this relation. In order to write the profile equations as a planar Hamiltonian 
system, one may just eliminate $u=j / \rho+\sigma$ and therefore obtain the second order differential equation on $\rho$ :

$$
-K(\rho) \rho^{\prime \prime}-\frac{1}{2} K(\rho)^{\prime} \rho^{\prime}+g_{0}(\rho)+\frac{j^{2}}{2 \rho^{2}}-\frac{1}{2} \sigma^{2}-q=0 .
$$

Recalling that

$$
\frac{\mathrm{d} F_{0}}{\mathrm{~d} \rho}=g_{0},
$$

equation 2.11] admits the obvious first integral

$$
-\frac{1}{2} K(\rho)\left(\rho^{\prime}\right)^{2}+F_{0}(\rho)-\frac{j^{2}}{2 \rho}-\left(\frac{1}{2} \sigma^{2}+q\right) \rho .
$$

The existence of homoclinic or heteroclinic orbits then depends on the parameters $\sigma, j$ and $q$ and on the behavior of the function $F_{0}$ - the function $K$ being only a matter of rescaling.

REMARK 1 Using the definition $j=\rho_{ \pm}\left(u_{ \pm}-\sigma\right)$ and recalling that $g_{0}=p_{0} v-\mathrm{d} p_{0} / \mathrm{d} v$, the equalities in 2.9 and 2.10) amount to requiring the equal area rule

$$
\int_{v_{-}}^{v_{+}}\left\{p_{0}(v)-p_{0}\left(v_{ \pm}\right)+\frac{p_{0}\left(v_{+}\right)-p_{0}\left(v_{-}\right)}{v_{-}-v_{+}}\left(v-v_{ \pm}\right)\right\} \mathrm{d} v=0 .
$$

This relation is of course possible only for a non-convex function $p_{0}$, for instance the van der Waals pressure law below critical temperature. In this case, $p_{0}$ is also non-monotone and the corresponding kink is a liquid-vapor interface. One may observe that equation $(2.12)$ is a generalization to dynamical phase boundaries of the well known Maxwell rule for equilibrium phase boundaries. In addition, for the van der Waals pressure law there is a range of temperatures, just above the critical temperature, for which $p_{0}$ is monotone and non-convex. Even though the corresponding kinks are no longer interfaces our subsequent analysis applies to them.

Unsurprisingly, the purely thermodynamical equation 2.12 also arises when one looks for traveling profiles of the Lagrangian model (1.5). We shall come back at the end of this section to the relation between Lagrangian traveling waves and Eulerian traveling waves. For the moment, we concentrate on the Lagrangian model (1.5), for which the discussion of traveling profiles is much simpler and more or less standard. The profile of a traveling wave solution of "speed" $\tau$-be careful that $\tau$ is not homogeneous in a velocity-of $(1.5)$ must satisfy the ODEs

$$
\left\{\begin{array}{l}
-\tau \dot{v}-\dot{u}=0, \\
-\tau \dot{u}+\dot{p_{0}}=-\left(\kappa \ddot{v}+\frac{1}{2} \dot{\kappa} \dot{v}\right)
\end{array}\right.
$$

where denotes the derivative with respect to $\zeta:=y-\tau t$. Eliminating $\dot{u}$ we obtain

$$
\kappa \ddot{v}+\frac{1}{2} \dot{\kappa} \dot{v}+p_{0}+\tau^{2} v \equiv \text { constant. }
$$

For a kink, we obtain the Rankine-Hugoniot conditions corresponding to discontinuous traveling wave solutions of the $p$-system:

$$
u_{+}+\tau v_{+}=u_{-}+\tau v_{-}, \quad p_{0}\left(v_{+}\right)-\tau u_{+}=p_{0}\left(v_{-}\right)-\tau u_{-},
$$


with their consequence

$$
\tau^{2}=\frac{p_{0}\left(v_{+}\right)-p_{0}\left(v_{-}\right)}{v_{-}-v_{+}},
$$

and the equal area rule 2.12 by multiplying the ODE

$$
\kappa(v) \ddot{v}+\frac{1}{2}(\kappa(v)) \ddot{v}=-p_{0}(v)+p_{0}\left(v_{ \pm}\right)-\tau^{2}\left(v-v_{ \pm}\right)
$$

by $\dot{v}$ and integrating once. A first integral of $(2.13)$ is indeed

$$
\frac{1}{2} \kappa(v) \dot{v}^{2}+I\left(v ; v_{ \pm}, \tau\right)
$$

with

$$
I\left(v ; v_{ \pm}, \tau\right):=f_{0}\left(v_{ \pm}\right)-f_{0}(v)-p_{0}\left(v_{ \pm}\right)\left(v-v_{ \pm}\right)+\frac{1}{2} \tau^{2}\left(v-v_{ \pm}\right)^{2} .
$$

This implies in particular that in the phase plane $\{(v, \dot{v})\}$ a connecting orbit to $\left(v_{ \pm}, 0\right)$ lies in the level set

$$
\left\{(v, \dot{v}) ; \frac{1}{2} \kappa(v) \dot{v}^{2}+I\left(v ; v_{ \pm}, \tau\right)=0\right\} .
$$

Before going further we need some assumptions on $p_{0}$ and $f_{0}$. We avoid too much generality and concentrate on assumptions that are met by the van der Waals pressure law.

- (Asymptotic behavior) The function $p_{0}=-\mathrm{d} f_{0} / \mathrm{d} v$ is smoothly defined on the interval $(b,+\infty)$ with $b>0$, and

$$
\lim _{v \rightarrow b} p_{0}(v)=+\infty, \quad \lim _{v \rightarrow+\infty} p_{0}(v)=0, \quad \lim _{v \rightarrow b} f_{0}(v)=+\infty, \quad \lim _{v \rightarrow+\infty} f_{0}(v)=-\infty .
$$

- (Monotonicity) The function $p_{0}$ is monotonically decaying except maybe on some interval where it is increasing.

- (Convexity) The function $p_{0}$ has at most two inflection points, in between which it is concave, and it is convex elsewhere.

These properties will be assumed without repetition in what follows. They imply in particular that any straight line of negative slope encounters the graph of $p_{0}$ at most four times.

One may rewrite (2.13) as a first order system by setting $w=\sqrt{\kappa} \dot{v}$, which yields

$$
\left\{\begin{array}{l}
\dot{v}=\frac{1}{\sqrt{\kappa}} w \\
\dot{w}=\frac{1}{\sqrt{\kappa}}\left(-p_{0}(v)+p_{0}\left(v_{ \pm}\right)-\tau^{2}\left(v-v_{ \pm}\right)\right) .
\end{array}\right.
$$

(The phase portrait of (2.14) being independent of the factor $1 / \sqrt{\kappa}$ we might assume here without loss of generality that $\kappa \equiv 1$.) An orbit of (2.14] necessarily lies on a level curve of the function

$$
(v, w) \mapsto \frac{1}{2} w^{2}+I\left(v ; v_{ \pm}, \tau\right) .
$$

Particular orbits are the fixed points $\left(v_{0}, 0\right)$ implicitly defined by

$$
p_{0}\left(v_{0}\right)+\tau^{2} v_{0}=p_{0}\left(v_{ \pm}\right)+\tau^{2} v_{ \pm}
$$


A geometrical interpretation of this equality is that $v_{0}$ is a point of intersection of the graph of $p_{0}$ with the so-called Rayleigh line, passing through $\left(v_{ \pm}, p_{0}\left(v_{ \pm}\right)\right)$and of slope $-\tau^{2}$. Because of our assumptions, the system (2.14) has at most four fixed points (of course including $\left(v_{ \pm}, 0\right)$ ). Their nature is easily determined. A fixed point $\left(v_{0}, 0\right)$ is a center if

$$
\frac{\mathrm{d} p_{0}}{\mathrm{~d} v}\left(v_{0}\right)>-\tau^{2}
$$

and a saddle if

$$
\frac{\mathrm{d} p_{0}}{\mathrm{~d} v}\left(v_{0}\right)<-\tau^{2} .
$$

Connecting orbits can therefore only be saddle-saddle connections. Furthermore, if $\left(v_{ \pm}, 0\right)$ is a saddle, a connecting orbit to this point must be included in

$$
\left\{(v, w) ; \frac{1}{2} w^{2}+I\left(v ; v_{ \pm}, \tau\right)=0\right\} .
$$

We thus see that $I\left(v ; v_{ \pm}, \tau\right)$ necessarily vanishes at point(s) where the orbit crosses the $v$-axis. Now the existence of zeroes of $I\left(v ; v_{ \pm}, \tau\right)$ can be investigated merely by the mean value theorem and the following observation. If $\left(v_{0}, p_{0}\left(v_{0}\right)\right)$ belongs to the Rayleigh line passing through $\left(v_{ \pm}, p_{0}\left(v_{ \pm}\right)\right)$ with slope $-\tau^{2}$ then

$$
I\left(v_{0} ; v_{ \pm}, \tau\right)=\int_{v_{ \pm}}^{v_{0}}\left(p(v)-\frac{1}{2}\left(p\left(v_{0}\right)+p\left(v_{ \pm}\right)\right)\right) \mathrm{d} v
$$

is a signed area between the graph of $p_{0}$ and the chord joining $v_{0}$ to $v_{ \pm}$. More precisely, assume that $\left(v_{0}, p\left(v_{0}\right)\right),\left(v_{1}, p\left(v_{1}\right)\right),\left(v_{2}, p\left(v_{2}\right)\right)$ are aligned on a Rayleigh line with slope $-\tau^{2}$, the graph of $p$ being below that line on the interval $\left(v_{0}, v_{1}\right)$ and above the line on $\left(v_{1}, v_{2}\right)$, with $v_{0}<v_{1}<v_{2}$. This means in particular that $I\left(\cdot ; v_{0}, \tau\right)$ and $I\left(\cdot ; v_{2}, \tau\right)$ are monotone on the intervals $\left(v_{0}, v_{1}\right)$ and $\left(v_{1}, v_{2}\right)$, and that $I\left(v_{1} ; v_{0}, \tau\right)<0$ and $I\left(v_{1} ; v_{2}, \tau\right)<0$. Additionally, from the planar dynamical system point of view, $\left(v_{0}, 0\right)$ and $\left(v_{2}, 0\right)$ are saddles, whereas $\left(v_{1}, 0\right)$ is a center of 2.14. When looking for connections to either $v_{0}$ or $v_{2}$ or both, one may observe that:

(i) if the signed area between the graph of $p$ and the Rayleigh line is negative, then $I\left(v_{2} ; v_{0}, \tau\right)<0$ and therefore $I\left(\cdot ; v_{0}, \tau\right)$ does not vanish on the interval $\left(v_{0}, v_{2}\right)$, whereas $I\left(v_{0} ; v_{2}, \tau\right)>0$, which implies that $I\left(\cdot ; v_{2}, \tau\right)$ vanishes exactly once on $\left(v_{0}, v_{2}\right)$, in fact on $\left(v_{0}, v_{1}\right)$ (see Figure 1 ;

(ii) if the signed area between the graph of $p$ and the Rayleigh line is equal to zero, then $I\left(v_{2} ; v_{0}, \tau\right)=I\left(v_{0} ; v_{2}, \tau\right)$ and neither $I\left(\cdot ; v_{0}, \tau\right)$ nor $I\left(\cdot ; v_{2}, \tau\right)$ vanish inside $\left(v_{0}, v_{2}\right)$;

(iii) if the signed area between the graph of $p$ and the Rayleigh line is positive, then $I\left(v_{0} ; v_{2}, \tau\right)<0$ and therefore $I\left(\cdot ; v_{2}, \tau\right)$ does not vanish on the interval $\left(v_{0}, v_{2}\right)$, whereas $I\left(v_{2} ; v_{0}, \tau\right)>0$, which implies that $I\left(\cdot ; v_{0}, \tau\right)$ vanishes exactly once on $\left(v_{0}, v_{2}\right)$, in fact on $\left(v_{1}, v_{2}\right)$.

From this we infer several kinds of connections. In case (i), there is a homoclinic connection to $v_{2}$. In the framework of van der Waals fluids below critical temperature this corresponds to a "mixed type" soliton propagating in the vapor phase. By mixed type we mean that unstable and maybe liquid states are present inside the bump. Similarly, in case (iii), there is a homoclinic connection to $v_{0}$, which corresponds for a van der Waals fluids below critical temperature to a "mixed type" soliton propagating in the liquid phase. Note that a similar connection exists in the absence of $v_{2}$, since $I\left(\cdot ; v_{0}, \tau\right)$ is increasingly tending to $+\infty$ at infinity. The critical case (ii) means that the equal area rule is satisfied and so there are heteroclinic connections from $v_{0}$ to $v_{2}$ and from 

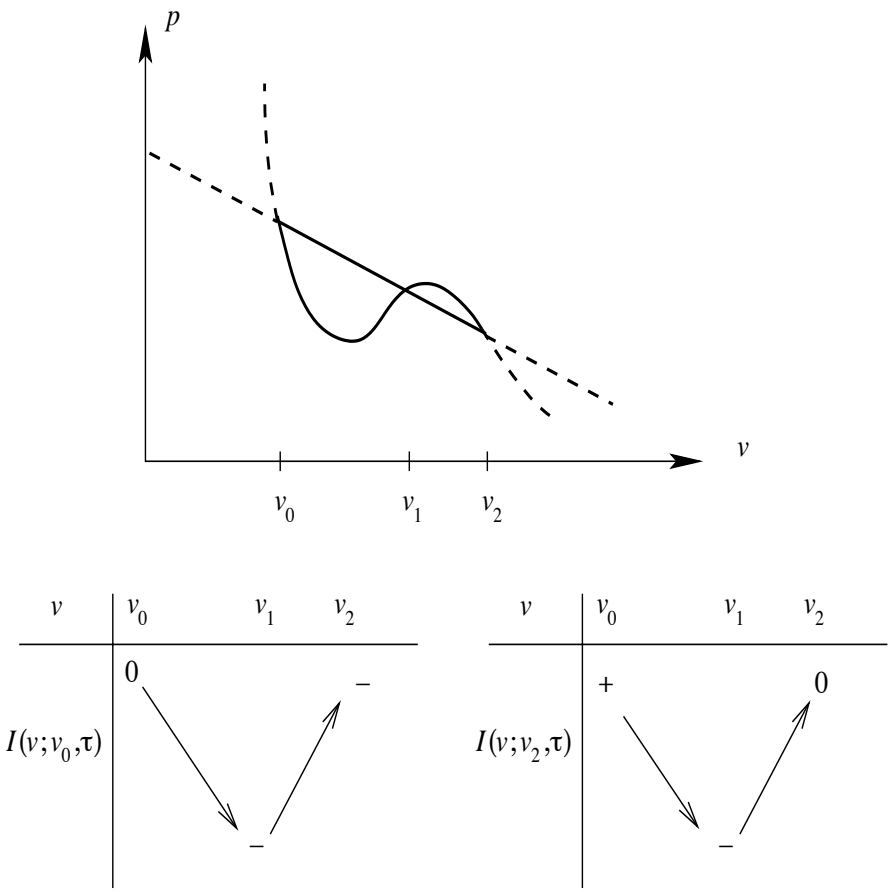

FIG. 1. Behavior of $I$ in case (i)

$v_{2}$ to $v_{0}$. For a van der Waals fluid below critical temperature this corresponds to what is called a diffuse liquid-vapor interface-by contrast with sharp interfaces, which correspond to discontinuous traveling wave solutions of the pure Euler equations.

Now, if there is a fourth point $v_{3}>v_{2}$ on the Rayleigh line, the solitons found in cases (i) and (iii) are not the only ones. For van der Waals fluids below critical temperature, this situation typically occurs when $v_{0}$ and $v_{2}$ are "close" to liquid and vapor equilibrium respectively, and $\tau^{2}$ is not too big, and more generally, for a given $v_{0}$, possibly far away from liquid equilibrium, there is always a finite range for $\tau^{2}$ for which the Rayleigh line intersects four times the graph of $p$. If this is the case, $I\left(\cdot ; v_{2}, \tau\right)$ equals 0 at $v_{2}$, is decaying on the interval $\left(v_{2}, v_{3}\right)$ and increasingly tending to $+\infty$ at infinity. This implies that it necessarily vanishes somewhere on the interval $\left(v_{3},+\infty\right)$ (see Figure 2). The phase portrait then shows that this zero necessarily corresponds to the intersection with the $v$-axis of a homoclinic connection to $v_{2}$. For a van der Waals fluid below critical temperature this corresponds to a pure vapor soliton. The same kind of soliton occurs when the line of slope $-\tau^{2}$ intersects the graph of $p$ only twice, at a saddle point $v_{2}$ and at a center point $v_{3}$ with $v_{2}<v_{3}$.

Finally, by the same argument we find that $I\left(\cdot ; v_{0}, \tau\right)$ vanishes on $\left(v_{3},+\infty\right)$ (see again Figure 2), yielding a homoclinic connection to $v_{0}$ provided that $I\left(\cdot ; v_{0}, \tau\right)$ does not vanish in between $v_{0}$ and $v_{3}$. For a van der Waals fluid below critical temperature this corresponds to a "huge mixed type" soliton, propagating in the liquid phase and containing both unstable states and vapor states inside its bump. The same kind of soliton occurs when the line of slope $-\tau^{2}$ intersects the graph of $p$ only twice but differently than above, at a saddle point $v_{0}$ (saddle) and at a center point $v_{3}$ with $v_{0}<v_{3}$. 

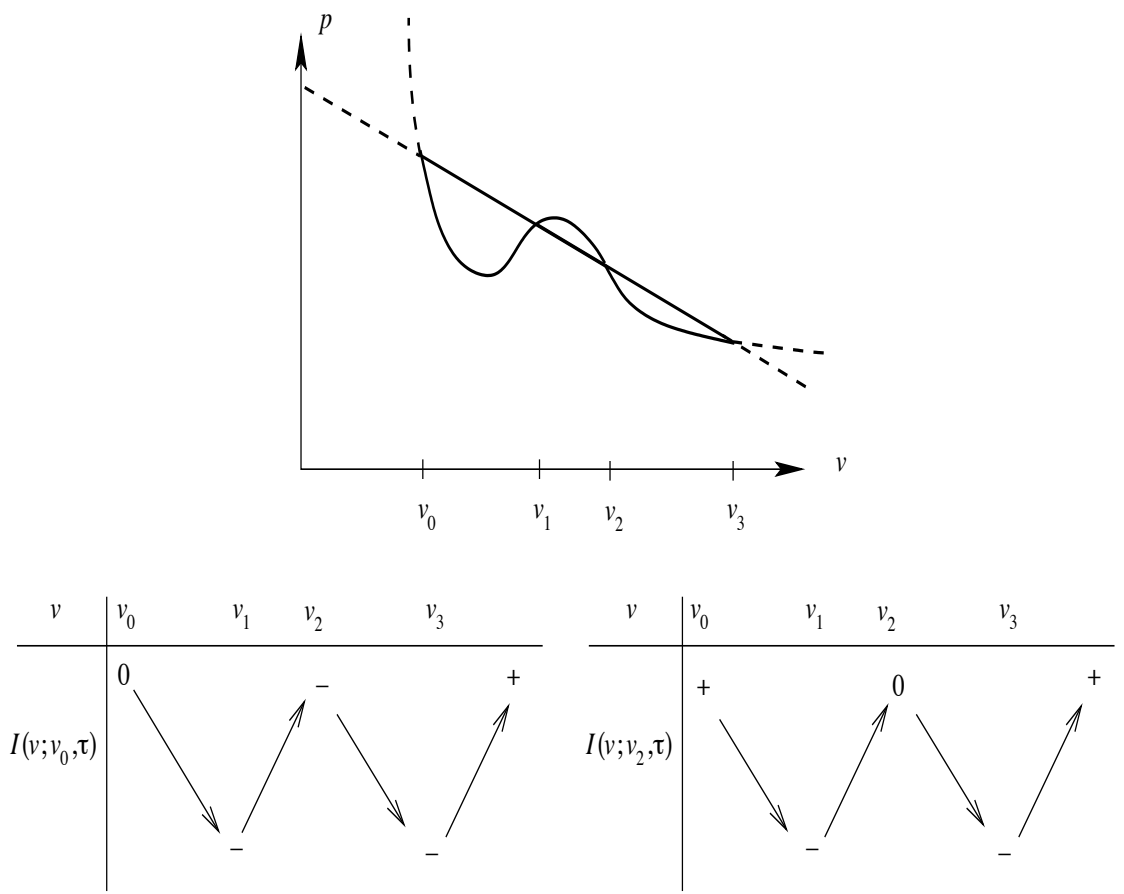

FIG. 2. Behavior of $I$ in the presence of a second center point $v_{3}$.

The three possible phase portraits corresponding to the three cases (i) (iii) are represented in Figures $3-5$ respectively, where the isothermals are obtained with the van dar Waals law for water (coefficients $a, b$ taken from [37]) at $T=600 \mathrm{~K}$.

PROPOSITION 2 (Classification of saddle-saddle connections) Let $S$ be a non-positive real number and consider the straight line $D_{S}$ of slope $S$ in the $(v, p)$ plane and define

$$
C_{S}:=\left\{v ;\left(v, p_{0}(v)\right\} \in D_{S}\right\} .
$$

Under our main assumptions on the functions $p_{0}$ and $f_{0}$,

$$
\text { either } C_{S}=\left\{v_{0}, v_{1}, v_{2}, v_{3}\right\} \quad \text { or } C_{S}=\left\{v_{0}, v_{1}\right\} \quad \text { or } C_{S}=\left\{v_{0}, v_{3}\right\} \quad \text { or } C_{S}=\left\{v_{2}, v_{3}\right\} \text {, }
$$

the points $v_{i}$ being ordered increasingly and whenever they exist

$$
\frac{\mathrm{d} p_{0}}{\mathrm{~d} v}\left(v_{0}\right)<S, \quad \frac{\mathrm{d} p_{0}}{\mathrm{~d} v}\left(v_{1}\right)>S, \quad \frac{\mathrm{d} p_{0}}{\mathrm{~d} v}\left(v_{2}\right)<S, \quad \frac{\mathrm{d} p_{0}}{\mathrm{~d} v}\left(v_{3}\right)>S,
$$

with $p_{0}$ being convex on $\left(v_{3},+\infty\right){ }^{1}$ Then the saddle-saddle connections of (2.14) belong to one of the following types, where we use a terminology referring to the van der Waals law below critical temperature 2

\footnotetext{
1 This makes the difference between the cases $C_{S}=\left\{v_{0}, v_{1}\right\}$ and $C_{S}=\left\{v_{0}, v_{3}\right\}$. This distinction is more for physical reasons than mathematical ones.

${ }^{2}$ In particular we mention in which phase the corresponding solitary waves are supposed to propagate.
} 

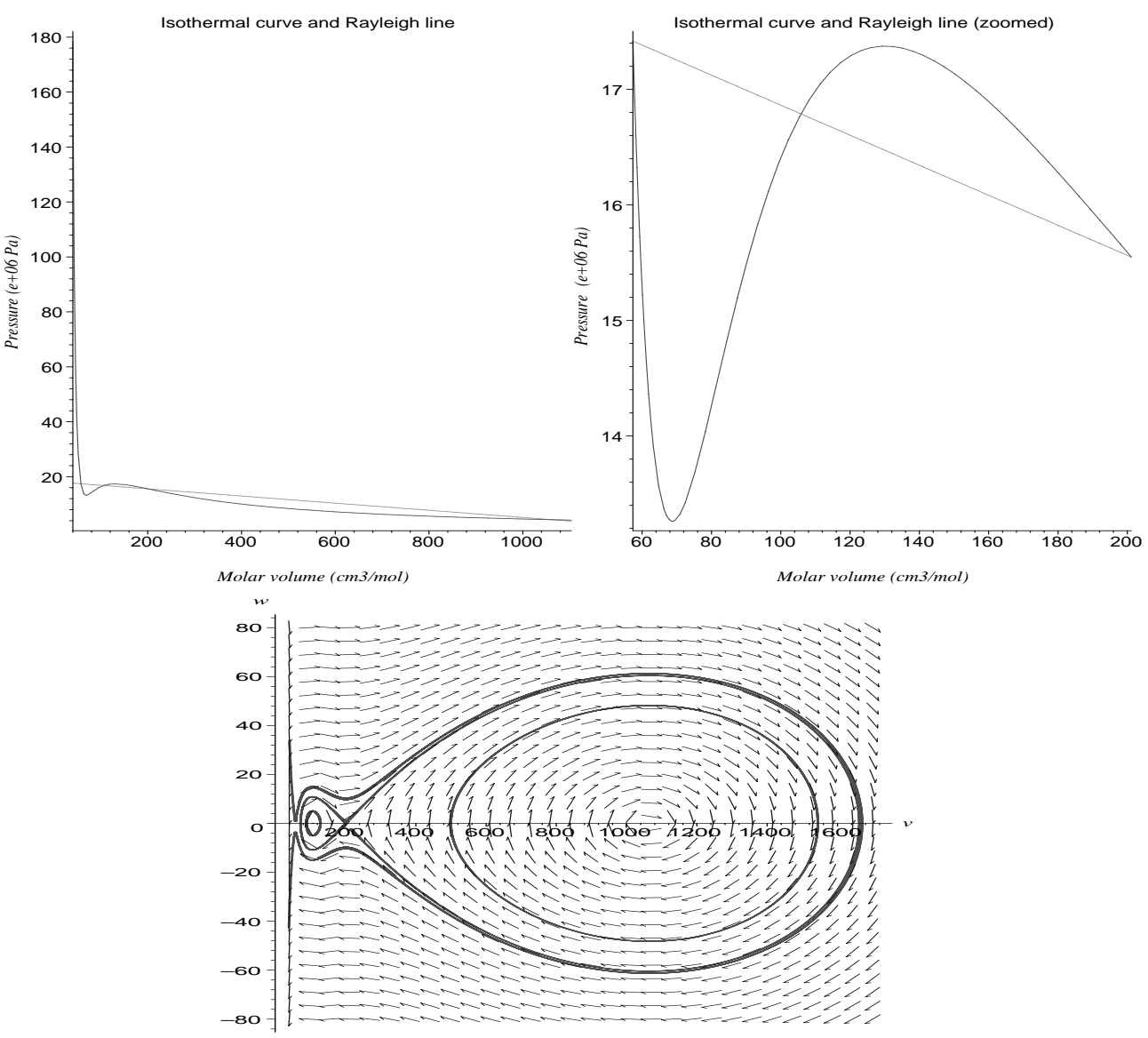

FIG. 3. Van der Waals isothermal curve, Rayleigh line and corresponding phase portrait in case (i)

(Temperate mixed vapor solitons) They correspond to orbits homoclinic to $v_{2}$ whose minimum is smaller than $v_{1}$. They exist if and only if

$$
\int_{v_{0}}^{v_{2}}\left(p(v)-\frac{1}{2}\left(p\left(v_{0}\right)+p\left(v_{2}\right)\right)\right) \mathrm{d} v<0 .
$$

(Kinks) They correspond to heteroclinic orbits whose endstates are $v_{0}$ and $v_{2}$. They exist if and only if

$$
\int_{v_{0}}^{v_{2}}\left(p(v)-\frac{1}{2}\left(p\left(v_{0}\right)+p\left(v_{2}\right)\right)\right) \mathrm{d} v=0 .
$$

These kinks are called diffuse interfaces when $p_{0}$ is increasing on some interval in between $v_{0}$ and $v_{2}$.

(Temperate mixed liquid solitons) They correspond to orbits homoclinic to $v_{0}$ whose maximum is greater than $v_{1}$ and smaller than $v_{2}$. They exist if and only if

$$
\int_{v_{0}}^{v_{2}}\left(p(v)-\frac{1}{2}\left(p\left(v_{0}\right)+p\left(v_{2}\right)\right)\right) \mathrm{d} v>0 .
$$



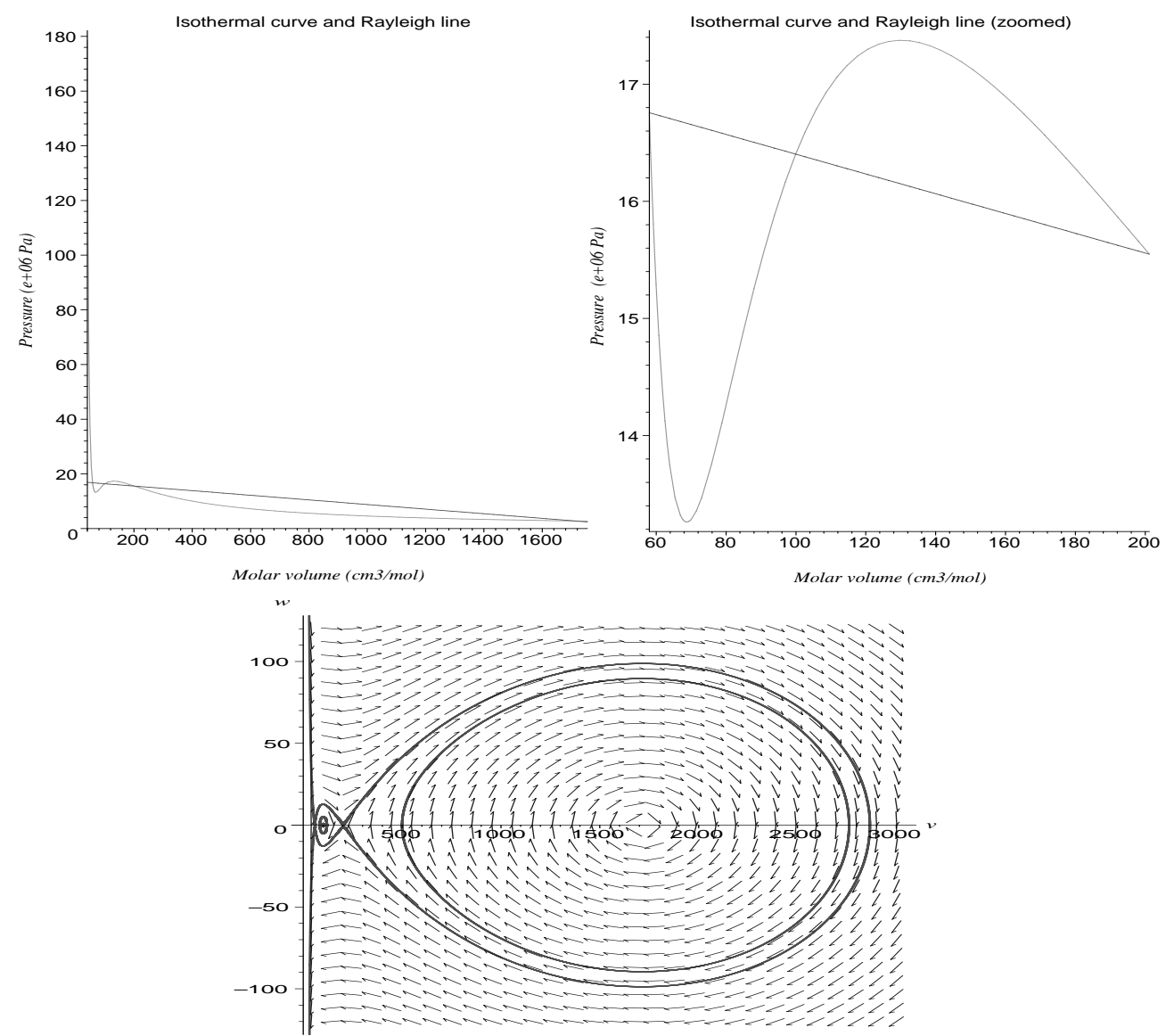

FIG. 4. Van der Waals isothermal curve, Rayleigh line and corresponding phase portrait in case (ii)

(Pure vapor solitons) They correspond to orbits homoclinic to $v_{2}$ whose maximum is greater than $v_{3}$. They exist as soon as $v_{2}$ and $v_{3}$ do.

(Large mixed liquid solitons) They correspond to orbits homoclinic to $v_{0}$ whose maximum is greater than $v_{3}$. They exist, if $v_{0}$ and $v_{3} \mathrm{do}$, in the absence of temperate mixed liquid solitons.

Lagrangian profiles/Eulerian profiles. All these connections have their counterparts as connections of the Eulerian equation 2.11, even though this is far from being obvious if looking directly at the profile equations (2.11) and (2.13). Recall indeed that the PDE model $(1.5)$ is obtained from (1.4) by using the differential relation $\mathrm{d} y=\rho \mathrm{d} x-\rho u \mathrm{~d} t$. If $(\rho, u)=(\rho(x-\sigma t), u(x-\sigma t))$ is a bounded traveling wave solution of 1.4 with $\rho \geqslant \rho>0$ everywhere, the associated Lagrangian mass coordinate reads $y=R-j t$, where $R$ is a primitive of $\rho$ with respect to $\xi:=x-\sigma t$ and $j=\rho(u-\sigma)$. Observing that the function $R$ is a diffeomorphism on $\mathbb{R}$, we obtain a traveling solution of "speed" $\tau:=-j$ of 1.5 merely by defining its profile as

$$
v:=\frac{1}{\rho \circ R^{-1}} .
$$



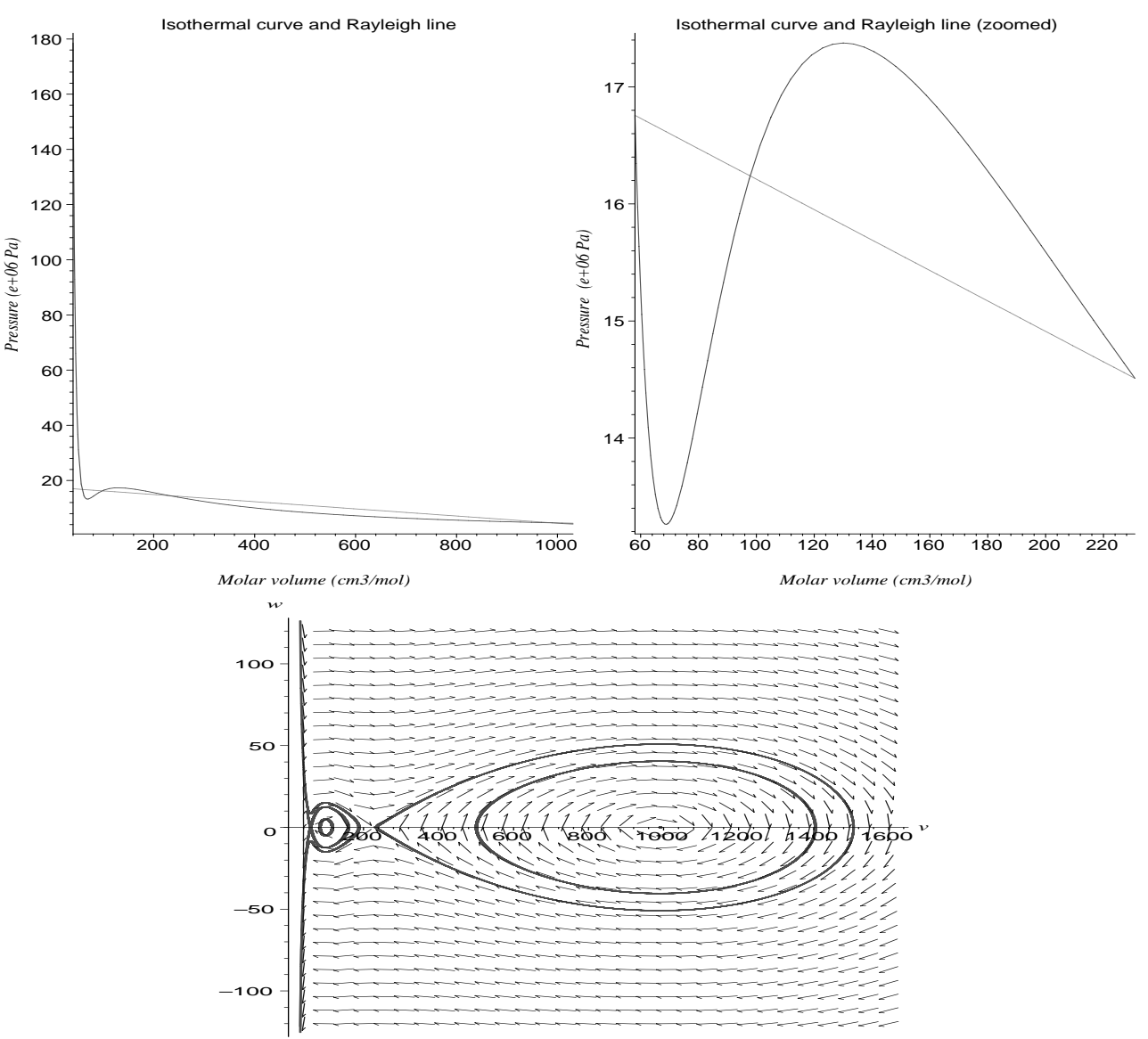

FIG. 5. Van der Waals isothermal curve, Rayleigh line and corresponding phase portrait in case (iii)

Conversely, if $(v, u)=(v(y-\tau t), u(y-\tau t))$ is a bounded traveling wave solution of 1.5 with $v \geqslant \underline{v}>0$ everywhere, we obtain a traveling solution of (1.5) with

$$
j:=-\tau, \quad \sigma:=u_{ \pm}-j v_{ \pm},
$$

by considering the differential equation

$$
R^{\prime}=\frac{1}{v(R)} .
$$

Since the function $v$ is bounded on $\mathbb{R}$, all solutions of this differential equation are global and form a one-parameter family of diffeomorphisms on $\mathbb{R}$. We thus obtain a one-parameter family of density profiles $\rho:=R^{\prime}$.

\section{Stability of kinks and solitons}

We are concerned here with the long time behavior of the isothermal models (1.4) and (1.5) when the initial data are close to either a kink or a soliton. The observability of such patterns is indeed closely related to that stability problem. 
The stability of solitary waves has been studied for more than a century. The first ideas certainly date back to the remarkable work of Boussinesq on the water waves in narrow channels [11], which appeared less than thirty years after John Scott Russel reported his famous observation, and twenty years before the work of Korteweg and de Vries. It is notable that (one of) equation(s) nowadays known as the Boussinesq equation takes the form of the system (1.5), with $\kappa$ constant and $p_{0}$ convex-in the most standard framework, $p_{0}(v)=v^{2}-v$. The stability analysis of solitary waves became very active in the 1970s and made a leap with the work of Grillakis, Shatah and Strauss, who derived a sharp stability criterion (S) [18]. As regards the Boussinesq equation, a class of solitons is known to satisfy $(\mathbf{S})$ in the case of a power-type law $\left(p_{0}(v)=v^{k}-v, k<5\right)$ [9]. We are basically interested in the same question for the different kinds of solitons with non-convex van der Waalstype laws. We shall present a reformulation of (S) enabling us to determine the cases in which it is satisfied.

As regards the stability of kinks, among which diffuse interfaces are of special interest, we do not know any work analogous to [9]. The spectral stability of kinks, which unlike solitons do not come in families parametrized by the wave speed, is known to be rather strong, in fact as much as we can hope for in a Hamiltonian framework [5]. However, this "neutral" spectral stability is far from being sufficient in itself to imply the non-linear stability of kinks. In this respect, we show below how the tools previously developed for solitons can be adapted and how far they can go in the stability analysis of kinks. Those tools crucially use the Hamiltonian structure of Korteweg models. Because of the translation invariance of these models, orbital stability is the best we can expect. It means that solutions stay in a given neighborhood of the one-dimensional manifold made of all shifted profiles, provided that they are close enough initially to either one of the profiles - this statement needs a functional framework that we shall specify later. In particular we do not expect any kind of control of the shift, unlike what is known for dissipative kinks [21].

For completeness, we shall deal with both systems (1.4) and (1.5), which can be recast in a similar Hamiltonian framework. As shown in Section 1, they can be rewritten in the abstract form

$$
\partial_{t} \mathbf{U}=\mathcal{J} \delta \mathcal{H}[\mathbf{U}]
$$

where $\mathcal{J}$ is a constant coefficients skewadjoint differential operator, and $\delta \mathcal{H}[\mathbf{U}]$ denotes the (formal) variational gradient of a functional

$$
\mathcal{H}[\mathbf{U}]=\int H(\mathbf{U}, \partial \mathbf{U})
$$

For the Eulerian system (1.4), $\partial$ stands for the derivative with respect to the space variable $x$ and we have

$$
\mathbf{U}=\left(\begin{array}{l}
\rho \\
u
\end{array}\right), \quad H(\mathbf{U}, \partial \mathbf{U})=\frac{1}{2} \rho u^{2}+F_{0}(\rho)+\frac{1}{2} K(\rho)\left(\partial_{x} \rho\right)^{2}, \quad \mathcal{J}=\left(\begin{array}{cc}
0 & -\partial_{x} \\
-\partial_{x} & 0
\end{array}\right)
$$

For the Lagrangian system 1.5, $\partial$ stands for the derivative with respect to the mass Lagrangian coordinate $y$ and we have

$$
\mathbf{U}=\left(\begin{array}{c}
v \\
u
\end{array}\right), \quad H(\mathbf{U}, \partial \mathbf{U})=\frac{1}{2} u^{2}+f_{0}(v)+\frac{1}{2} \kappa(v)\left(\partial_{y} v\right)^{2}, \quad \mathcal{J}=\left(\begin{array}{cc}
0 & \partial_{y} \\
\partial_{y} & 0
\end{array}\right)
$$


The definition of $\mathcal{H}$ is only formal here because $H(\mathbf{U}, \partial \mathbf{U})$ is not integrable, as far as we are concerned. However, for functions $\mathbf{U}$ that coincide asymptotically with a reference solution $\underline{\mathbf{U}}$ at $\pm \infty$

$$
\mathcal{H}[\mathbf{U} ; \underline{\mathbf{U}}]:=\int(H(\mathbf{U}, \partial \mathbf{\mathbf { U }})-H(\underline{\mathbf{U}}, \partial \underline{\mathbf{U}}))
$$

does make sense provided that the decay of $\mathbf{U}-\underline{\mathbf{U}}$ is sufficiently fast at $\pm \infty$. In this case the variational gradient of $\mathcal{H}$ with respect to $\mathbf{U}$ is independent of $\underline{\mathbf{U}}$ and given as usual by

$$
\int \delta \mathcal{H}[\mathbf{U}] \cdot \dot{\mathbf{U}}=\left.\frac{\mathrm{d}}{\mathrm{d} \theta} \mathcal{H}[\mathbf{U}+\theta \dot{\mathbf{U}} ; \underline{\mathbf{U}}]\right|_{\theta=0}=\int \mathrm{d} H(\mathbf{U}, \partial \mathbf{U}) \cdot(\dot{\mathbf{U}}, \partial \dot{\mathbf{U}}),
$$

for all $\dot{\mathbf{U}} \in \mathcal{D}(\mathbb{R})$. Recall that for the Eulerian system [1.4,

$$
\delta \mathcal{H}[\mathbf{U}]=\left(\begin{array}{l}
\frac{1}{2} u^{2}+g_{0}(\rho)-K(\rho) \partial^{2} \rho-\frac{1}{2} \frac{\mathrm{d} K}{\mathrm{~d} \rho}(\rho)(\partial \rho)^{2} \\
\rho u
\end{array}\right), \quad \mathbf{U}=\left(\begin{array}{l}
\rho \\
u
\end{array}\right),
$$

while for the system $(1.5)$,

$$
\delta \mathcal{H}[\mathbf{U}]=\left(\begin{array}{l}
-p_{0}(v)-\kappa(v) \partial^{2} v-\frac{1}{2} \frac{\mathrm{d} \kappa}{\mathrm{d} v}(v)(\partial v)^{2} \\
u
\end{array}\right), \quad \mathbf{U}=\left(\begin{array}{l}
v \\
u
\end{array}\right) .
$$

\subsection{Variational approach to traveling profiles}

We are interested in traveling wave solutions of either (1.4) or [1.5), and more specifically those which achieve finite limits at $\pm \infty$. Using the formalism above, these traveling waves appear to correspond to critical points of the functional $\mathcal{H}$ under the three constraints

$$
\int U_{1}, \quad \int U_{2}, \quad \int U_{1} U_{2}=\text { constant, }
$$

where $U_{1}$ and $U_{2}$ stand for the components of $\mathbf{U}$. Again this is at first glance only formal because $U_{1}, U_{2}$ and $U_{1} U_{2}$ are a priori not integrable. These "functionals" nevertheless correspond to first integrals of the system 3.15 in the sense explained below.

First integrals of 3.15. The Hamiltonian $H$ corresponds formally to a first integral of 3.15. Let us look for a suitable functional framework in which this statement makes sense. Recalling the special form of the Hamiltonian

$$
H(\mathbf{U}, \partial \mathbf{U})=H_{0}(\mathbf{U})+\frac{1}{2} k\left(U_{1}\right)\left(\partial U_{1}\right)^{2},
$$

which holds true for both (1.4) and (1.5) - with either $k=K$ or $k=\kappa$-we find that for a (classical) solution $\mathbf{U}$ of 3.15 ,

$$
\begin{aligned}
\int_{a}^{b} H\left(\mathbf{U}(z, t), \partial_{z} \mathbf{U}(z, t)\right) \mathrm{d} z=\int_{a}^{b} H\left(\mathbf{U}(z, 0), \partial_{z} \mathbf{U}(z, 0)\right) \mathrm{d} z+\int_{0}^{t} \int_{a}^{b} \delta \mathcal{H}[U] \cdot \mathcal{J} \delta \mathcal{H}[U] \\
+\int_{0}^{t}\left(k\left(U_{1}(b, \tau)\right) \partial_{z} U_{1}(b, \tau) \partial_{t} U_{1}(b, \tau)-k\left(U_{1}(a, \tau)\right) \partial_{z} U_{1}(a, \tau) \partial_{t} U_{1}(a, \tau)\right) \mathrm{d} \tau
\end{aligned}
$$


for all $t>0$ and $a<b$. Now recalling the special form of the operator

$$
\mathcal{J}=\mathbf{J}_{\varepsilon} \partial, \quad \mathbf{J}_{\varepsilon}:=\varepsilon\left(\begin{array}{ll}
0 & 1 \\
1 & 0
\end{array}\right), \quad \varepsilon \in\{-1,1\}
$$

(with $\varepsilon=-1$ for (1.4) and $\varepsilon=1$ for (1.5), we may rewrite the previous equality as

$$
\begin{aligned}
\int_{a}^{b} H\left(\mathbf{U}(z, t), \partial_{z} \mathbf{U}(z, t)\right) \mathrm{d} z=\int_{a}^{b} H\left(\mathbf{U}(z, 0), \partial_{z} \mathbf{U}(z, 0)\right) \mathrm{d} z \\
+\frac{1}{2} \int_{0}^{t}\left(\delta \mathcal{H}[U] \cdot \mathbf{J}_{\varepsilon} \delta \mathcal{H}[U]\right)(b, \tau)-\left(\delta \mathcal{H}[U] \cdot \mathbf{J}_{\varepsilon} \delta \mathcal{H}[U]\right)(a, \tau) \mathrm{d} \tau \\
+\int_{0}^{t}\left(k\left(U_{1}(b, \tau)\right) \partial_{z} U_{1}(b, \tau) \partial_{t} U_{1}(b, \tau)-k\left(U_{1}(a, \tau)\right) \partial_{z} U_{1}(a, \tau) \partial_{t} U_{1}(a, \tau)\right) \mathrm{d} \tau
\end{aligned}
$$

Finally, using the specific form of $H$ and $\mathcal{J}$, the time derivatives $\partial_{t} U_{1}$ are easily replaced by "spatial" derivatives. More precisely, for the Lagrangian system (1.5) we have $\partial_{t} U_{1}=\partial_{y} U_{2}$, and for the Eulerian system (1.4) we have $\partial_{t} U_{1}=-\partial_{x}\left(U_{1} U_{2}\right)$. This leads to the following.

LEMma 1 Assume that $\mathbf{U}$ and $\widehat{\mathbf{U}}$ are two smooth solutions of the abstract version (3.15) of either (1.4) or [1.5), that $\widehat{\mathbf{U}}$ belongs to $\mathcal{C}\left([0, T] ; W^{2, \infty}\right)$ and

$$
\lim _{z \rightarrow \pm \infty}(\mathbf{U}-\widehat{\mathbf{U}})(z, t)=0, \quad \lim _{z \rightarrow \pm \infty} \partial_{z}(\mathbf{U}-\widehat{\mathbf{U}})(z, t)=0, \quad \lim _{z \rightarrow \pm \infty} \partial_{z z}^{2}\left(U_{1}-\widehat{U}_{1}\right)(z, t)=0
$$

for all $t \in[0, T]$. Then, if the integral

$$
\int_{-\infty}^{+\infty}(H[\mathbf{U}]-H[\widehat{\mathbf{U}}])(z, t) \mathrm{d} z
$$

is convergent at time $t=0$, it remains convergent and in fact is constant for all time $t \in[0, T]$.

Proof. We just make the difference between the equality obtained above for $\mathbf{U}$ and its analogue for $\widehat{\mathbf{U}}$, and let $a$ go to $-\infty$, and $b$ go to $+\infty$.

Note that the asymptotic behaviors requested hold true in particular if $\mathbf{U}-\widehat{\mathbf{U}}$ belongs to $\mathcal{C}\left([0, T] ; H^{3} \times H^{2}\right)$. For the Lagrangian system [1.5], one may relax the assumption on the first derivative to

$$
\lim _{y \rightarrow \pm \infty} \partial_{y}\left(U_{1}-\widehat{U}_{1}\right)(y, t)=0 .
$$

As regards the "functionals" $\int U_{1}, \int U_{2}$ and $\int U_{1} U_{2}$, they are also formal first integrals of (3.15), in the sense that integrands satisfy conservation laws, which is obvious for the first two of them. The third conservation law has to do with the invariance of 3.15 under spatial translations, as explained in [4. pp. 8-9] in connection with Olver's generalized version of Noether's theorem. Indeed, $U_{1} U_{2}$ is what Benjamin calls an impulse, since the variational gradient of $\mathcal{Q}[\mathbf{U}]=\int U_{1} U_{2}$ is such that $\mathcal{J} \delta \mathcal{Q}[\mathbf{U}]$ is—up to a harmless \pm sign—equal to $\partial \mathbf{U}$. We can also compute directly the conservation law of $U_{1} U_{2}$. For any smooth solution of 3.15,

$$
\partial_{t}\left(U_{1} U_{2}\right)=U_{2} \partial_{t} U_{1}+U_{1} \partial_{t} U_{2}=\varepsilon \mathbf{J}_{\varepsilon} \mathbf{U} \cdot \partial_{t} \mathbf{U}=\varepsilon \mathbf{J}_{\varepsilon} \mathbf{U} \cdot \partial_{z} \mathbf{J}_{\varepsilon} \delta \mathcal{H}[\mathbf{U}]=\varepsilon \mathbf{U} \cdot \partial_{z} \delta \mathcal{H}[\mathbf{U}]
$$

because of the symmetry of $\mathbf{J}_{\varepsilon}$ and the property $\mathbf{J}_{\varepsilon}^{2}=\mathbf{I}$. The fact that $\mathbf{U} \cdot \partial_{z} \delta \mathcal{H}[\mathbf{U}]$ is an exact derivative is then easily verified in both the Lagrangian and the Eulerian framework. For, the 
contribution of $H_{0}$ to $H$ corresponds to either the so-called $p$-system-obtained from (1.5) by setting $\kappa \equiv 0$ - or the Euler system obtained from (1.4) by setting $K \equiv 0$, which are known to admit $U_{1} U_{2}$ as a mathematical entropy, and the contribution of capillarity is of the same form in both frameworks. In the Lagrangian framework for instance

$$
\mathbf{U} \cdot \partial_{y} \delta \mathcal{H}[\mathbf{U}]=\partial_{y}\left(\frac{1}{2} u^{2}-q_{0}(v)-v \kappa(v) \partial_{y y}^{2} v+\frac{1}{2}\left(\kappa(v)-v \frac{\mathrm{d} \kappa}{\mathrm{d} v}(v)\right)\left(\partial_{y} v\right)^{2}\right),
$$

where $\mathrm{d} q_{0} / \mathrm{d} v=v \mathrm{~d} p_{0} / \mathrm{d} v$. Now, by the same argument as in Lemma1, we show the following.

LEMmA 2 In the framework of Lemma1, if the integral

$$
\int_{-\infty}^{+\infty}(\mathbf{U}-\widehat{\mathbf{U}})(z, t) \mathrm{d} z
$$

is convergent at time $t=0$, it remains convergent and in fact is constant for all time $t \in[0, T]$. And the same is true for

$$
\int_{-\infty}^{+\infty}\left(U_{1} U_{2}-\widehat{U}_{1} \widehat{U}_{2}\right)(z, t) \mathrm{d} z
$$

Equation of connecting profiles. We refer to Section 2 for the explicit equations governing the connecting profiles of both systems (1.4) and (1.5). Here we show how those equations read in the abstract setting [3.15, in order to justify our claim, generalizing the result pointed out in [4, p. 11] that traveling profiles are critical points of $\mathcal{H}$ under constraints. The profile of a traveling wave solution $\mathbf{U}=\mathbf{U}(z-\sigma t)$ of 3.15 ) clearly satisfies the ODE

$$
\mathbf{J}_{\varepsilon} \partial \delta \mathcal{H}[\mathbf{U}]+\sigma \partial \mathbf{U}=0 .
$$

Using again the fact that $\mathbf{J}_{\varepsilon}^{2}$ is the identity $2 \times 2$ matrix, this is equivalent to

$$
\delta \mathcal{H}[\mathbf{U}]+\sigma \mathbf{J}_{\varepsilon} \mathbf{U} \equiv \text { constant } .
$$

In [4] the constant is equal to 0 because only solitons vanishing at $\infty$ are considered, with a Hamiltonian satisfying $\delta \mathcal{H}[0]=0$. Here we are interested in connecting profiles with non-zero, possibly different endstates, which do not cancel $\delta \mathcal{H}$. So the constant is generically non-zero, and its components can be seen as Lagrange multipliers associated with the constraints $\int U_{1}$ and $\int U_{2}$ respectively. Similarly to [4], $-\varepsilon \sigma$ can be seen as a Lagrange multiplier associated with the constraint $\int U_{1} U_{2}$, of which the variational gradient is precisely $\varepsilon \mathbf{J}_{\varepsilon} \mathbf{U}$.

From now on, we fix a saddle-saddle connecting profile $\underline{\mathbf{U}}$ of either (1.4) or (1.5), which can be either a soliton (i.e. a homoclinic connection, the endstates being $\mathbf{U}_{-}=\mathbf{U}_{+}$) or a kink (i.e. a heteroclinic connection, the endstates $\mathbf{U}_{-}$and $\mathbf{U}_{+}$being different), obtained in the framework of Proposition 2 There are crucial qualitative differences between solitons and kinks:

(1) kinks are monotone (in the sense that both components of $\underline{\mathbf{U}}$ are monotone, or equivalently the components of $\partial \underline{\mathbf{U}}$ have constant sign along the real line) whereas solitons are not (they are symmetric with respect to the point where they achieve their extremum),

(2) solitons come in families parametrized by the wave speed whereas kinks are fully determined by either one of their endstates. 
In any case, the set of profiles connecting the endstates $\mathbf{U}_{ \pm}$with wave speed $\sigma$ is made of all translates of $\underline{\mathbf{U}}$. We shall use repeatedly the simplified notation $\underline{\mathbf{U}}_{s}=\underline{\mathbf{U}}(\cdot+s)$ for these translates. We shall refer to $\underline{\mathbf{U}}$ as a capillary profile. By construction, $\underline{\mathbf{U}}$ is associated with a traveling wave solution $t \mapsto \widehat{\mathbf{U}}(\cdot, t)=\underline{\mathbf{U}}(\cdot-\sigma t)$ of the corresponding Hamiltonian system (3.15). Our purpose is to investigate the stability of $\widehat{\mathbf{U}}$, that is, the long time behavior of solutions of 3.15 that are close to $\underline{\mathbf{U}}$ initially.

We shall begin with the spectral stability analysis, including the stability criterion of Grillakis et al. and then turn to the non-linear stability issue. Despite the better stability of kinks from the variational point of view-a fact related to the observations in (1) and (2) here above, the stability of kinks at the non-linear level is to some extent poorer than the one of solitons.

\subsection{Spectral stability of capillary profiles}

As far as possible, we shall perform the stability analysis on the abstract level, which mostly avoids technical calculations and deals simultaneously with the Eulerian model (1.4) and its Lagrangian counterpart 1.5. Even though the stability results should in fine be the same for physical reasons, we think indeed interesting to study both systems, in particular because of their relationship with other physical frameworks: water waves for (1.5) and Quantum Hydrodynamics for (1.4).

The first, natural approach to study the stability of a traveling wave $\widehat{\mathbf{U}}$ is to linearize $\sqrt{3.15}$ about $\widehat{\mathbf{U}}$ in a "Galilean frame' 1 attached to $\widehat{\mathbf{U}}$, in which $\widehat{\mathbf{U}}$ becomes stationary. We obtain a system of the form

$$
\partial_{t} \mathbf{V}=L \mathbf{V},
$$

where $L$ is a third order differential operator in the "spatial" variable. As regards kinks, it was shown in [5] that the spectrum of $L$ coincides with the imaginary axis. This neutral stability of $\widehat{\mathbf{U}}$ is the best we can hope for in a Hamiltonian framework, and prevents us from using the refined Green's function techniques that have been successfully applied to traveling waves in dissipative models [40, 20, 21, 38]. by

In order to use Hamiltonian tools, consider the Hessian of $\mathcal{H}$ at $\underline{\mathbf{U}}$, which is the operator defined

$$
\int \dot{\mathbf{U}}^{\mathrm{t}} \operatorname{Hess} \mathcal{H}[\underline{\mathbf{U}}] \dot{\mathbf{U}}=\frac{\mathrm{d}^{2}}{\mathrm{~d} \theta^{2}} \mathcal{H}[\underline{\mathbf{U}}+\theta \dot{\mathbf{U}}]_{\mid \theta=0}=\int \frac{\mathrm{d}^{2}}{\mathrm{~d} \theta^{2}} H(\underline{\mathbf{U}}+\theta \dot{\mathbf{U}}, \partial \underline{\mathbf{U}}+\theta \partial \dot{\mathbf{U}})_{\mid \theta=0}
$$

for all $\dot{\mathbf{U}} \in \mathcal{D}(\mathbb{R})$. Since the functional $\mathcal{H}$ splits as

$$
\mathcal{H}[\mathbf{U}]=\mathcal{H}_{0}[\mathbf{U}]+\mathcal{K}\left[U_{1}\right], \quad \mathcal{K}[U]:=\int \frac{1}{2} k(U)(\partial U)^{2},
$$

we have

$$
\text { Hess } \mathcal{H}[\underline{\mathbf{U}}]=\operatorname{Hess} \mathcal{H}_{0}[\underline{\mathbf{U}}]+\operatorname{Hess} \mathcal{K}\left[\underline{U}_{1}\right]
$$

with Hess $\mathcal{K}\left[\underline{U}_{1}\right]$ being a Sturm-Liouville operator. More precisely, in the Eulerian framework, we have

$$
\operatorname{Hess} \mathcal{H}[\underline{\mathbf{U}}]=\left(\begin{array}{cc}
\frac{\mathcal{M}}{\underline{u}} & \underline{\underline{\rho}}
\end{array}\right), \quad \underline{\mathcal{M}}=-\partial_{x} K(\underline{\rho}) \partial_{x}+\frac{\mathrm{d} g_{0}}{\mathrm{~d} \rho}(\underline{\rho})-\frac{\mathrm{d} K}{\mathrm{~d} \rho}(\underline{\rho}) \partial_{x x}^{2} \underline{\rho}-\frac{1}{2} \frac{\mathrm{d}^{2} K}{\mathrm{~d} \rho^{2}}(\underline{\rho})\left(\partial_{x} \underline{\rho}\right)^{2},
$$

1 The term Galilean frame is in fact meaningful only for the Eulerian model 1.4 . 
while in the Lagrangian framework

$$
\operatorname{Hess} \mathcal{H}[\underline{\mathbf{U}}]=\left(\begin{array}{cc}
\frac{\mathcal{M}}{0} & 0 \\
0 & 1
\end{array}\right), \quad \underline{\mathcal{M}}=-\partial_{y} \kappa(\underline{v}) \partial_{y}-\frac{\mathrm{d} p_{0}}{\mathrm{~d} v}(\underline{v})-\frac{\mathrm{d} \kappa}{\mathrm{d} v}(\underline{v}) \partial_{y y}^{2} \underline{v}-\frac{1}{2} \frac{\mathrm{d}^{2} \kappa}{\mathrm{d} v^{2}}(\underline{v})\left(\partial_{y} \underline{v}\right)^{2} .
$$

In fact, since the profile $\underline{\mathbf{U}}$ is a critical point of $\mathcal{H}$ under constraints, we also have to take into account the Hessian of the quadratic functional $\int U_{1} U_{2}$, which is the constant operator $\varepsilon \mathbf{J}_{\varepsilon}$, and study the possible monotonicity of the operator

$$
\mathcal{L}:=\operatorname{Hess} \mathcal{H}[\underline{\mathbf{U}}]+\sigma \mathbf{J}_{\varepsilon} .
$$

The properties of $\mathcal{L}$ of course depend on the nature of the connecting orbit $\underline{\mathbf{U}}$. This is where the differences between kinks and solitons play a crucial role. We say $\underline{\mathbf{U}}$ is monotone if its first component $\underline{U}_{1}$ is monotone-which by the Rankine-Hugoniot relation also implies that $\underline{U}_{2}$ is monotone. We call the endstates $\mathbf{U}_{ \pm}$subsonic if both inequalities

$$
\left|\frac{p\left(v_{+}\right)-p\left(v_{-}\right)}{v_{+}-v_{-}}\right|<\left|\frac{\mathrm{d} p}{\mathrm{~d} v}\left(v_{ \pm}\right)\right|
$$

are satisfied. From the dynamical systems point of view this means that $\underline{\mathbf{U}}$ is a saddle-saddle connection. See Section 2

LEMma 3 Consider the ODE governing the traveling wave solutions of either (1.4) or 1.5, written in the abstract form:

$$
\mathbf{J}_{\varepsilon} \partial \delta \mathcal{H}[\mathbf{U}]+\sigma \partial \mathbf{U}=0,
$$

and $\underline{\mathbf{U}}$ a saddle-saddle connection of the integrated ODE

$$
\delta \mathcal{H}[\mathbf{U}]+\sigma \mathbf{J}_{\varepsilon} \mathbf{U} \equiv \delta \mathcal{H}\left[\mathbf{U}_{ \pm}\right]+\sigma \mathbf{J}_{\varepsilon} \mathbf{U}_{ \pm} .
$$

If $\underline{\mathbf{U}}$ is monotone then the operator

$$
\mathcal{L}:=\operatorname{Hess} \mathcal{H}[\underline{\mathbf{U}}]+\sigma \mathbf{J}_{\varepsilon}
$$

is monotone, i.e.

$$
\int \dot{\mathbf{U}}^{\mathrm{t}} \mathcal{L} \cdot \dot{\mathbf{U}} \geqslant 0
$$

for all $\dot{\mathbf{U}} \in \mathcal{D}(\mathbb{R})$. Furthermore, if $\int \dot{U}_{1} \partial \underline{U}_{1}=0$ and $\dot{\mathbf{U}} \neq 0$ then

$$
\int \dot{\mathbf{U}}^{\mathrm{t}} \mathcal{L} \cdot \dot{\mathbf{U}}>0
$$

Proof. We start with the Eulerian framework-the Lagrangian one being simpler. We recall that

$$
\mathcal{L}=\left(\begin{array}{cc}
\underline{\mathcal{M}} & \underline{u}-\sigma \\
\underline{u}-\sigma & \underline{\rho}
\end{array}\right), \quad \underline{\mathcal{M}}=-\partial K(\underline{\rho}) \partial+\frac{\mathrm{d} g_{0}}{\mathrm{~d} \rho}(\underline{\rho})-\frac{\mathrm{d} K}{\mathrm{~d} \rho}(\underline{\rho}) \partial^{2} \underline{\rho}-\frac{1}{2} \frac{\mathrm{d}^{2} K}{\mathrm{~d} \rho^{2}}(\underline{\rho})(\partial \underline{\rho})^{2} .
$$

So the monotonicity of $\mathcal{L}$ depends upon the monotonicity of the Sturm-Liouville operator $\mathcal{M}:=$ $\underline{\mathcal{M}}-(\underline{u}-\sigma)^{2} / \rho$. In fact, this operator $\mathcal{M}$ was already shown to be monotone in [5], Proposition 1$, p. 243$]$. We briefly recall the arguments. First of all, the subsonicity of endstates implies that the 
essential spectrum of $\mathcal{M}$ is positive, bounded away from 0 . Secondly, 0 is an eigenvalue of $\mathcal{M}$ with associated eigenfunction $\partial \rho$ - this is due to the translation invariance. Indeed, we can see directly from the ODE

$$
\partial \delta \mathcal{H}[\underline{\mathbf{U}}]+\sigma \mathbf{J}_{-1} \partial \underline{\mathbf{U}}=0
$$

that $\mathcal{L} \cdot \partial \underline{\mathbf{U}}=0$, by definition of $\mathcal{L}$, using the fact that $\partial \delta \mathcal{H}[\underline{\mathbf{U}}]=\operatorname{Hess} \mathcal{H}[\underline{\mathbf{U}}] \cdot \partial \underline{\mathbf{U}}$. And this readily implies that $\mathcal{M} \cdot \partial \rho=0$. Since $\partial \rho$ has a constant sign by assumption, the standard theory of SturmLiouville operators [32, 35] shows that 0 must be the lowest eigenvalue of $\mathcal{M}$, which yields the monotonicity of $\mathcal{M}$. Furthermore, there exists $c>0$ so that for $\int \dot{\rho} \partial \underline{\rho}=0$,

$$
\int \dot{\rho} \mathcal{M} \cdot \dot{\rho} \geqslant c\|\dot{\rho}\|_{L^{2}(\mathbb{R})}^{2}
$$

and thus

$$
\int \dot{\mathbf{U}}^{\mathrm{t}} \mathcal{L} \cdot \dot{\mathbf{U}} \geqslant c\|\dot{\rho}\|_{L^{2}(\mathbb{R})}^{2}+\left\|\frac{\rho \dot{u}+(\underline{u}-\sigma) \dot{\rho}}{\sqrt{\underline{\rho}}}\right\|_{L^{2}(\mathbb{R})}^{2} .
$$

The same arguments work in the Lagrangian framework. The Sturm-Liouville operator to be considered is

$$
\mathcal{M}=\underline{\mathcal{M}}-\sigma^{2}=-\partial \kappa(\underline{v}) \partial-\frac{\mathrm{d} p_{0}}{\mathrm{~d} v}(\underline{v})-\sigma^{2}-\frac{\mathrm{d} \kappa}{\mathrm{d} v}(\underline{v}) \partial^{2} \underline{v}-\frac{1}{2} \frac{\mathrm{d}^{2} \kappa}{\mathrm{d} v^{2}}(\underline{v})(\partial \underline{v})^{2}
$$

and it is monotone for the same reasons as before. The final estimate looks simpler:

$$
\int \dot{\mathbf{U}}^{\mathrm{t}} \mathcal{L} \cdot \dot{\mathbf{U}} \geqslant c\|\dot{v}\|_{L^{2}(\mathbb{R})}^{2}+\|\dot{u}+\sigma \dot{v}\|_{L^{2}(\mathbb{R})}^{2}
$$

for $\int \dot{v} \partial \underline{v}=0$.

REMARK 2 We may improve the above estimate

$$
\int \dot{U} \mathcal{M} \cdot \dot{U} \geqslant c\|\dot{U}\|_{L^{2}(\mathbb{R})}^{2}
$$

(omitting the subscript 1 for simplicity) to

$$
\int \dot{U} \mathcal{M} \cdot \dot{U} \geqslant c^{\prime}\|\dot{U}\|_{H^{1}(\mathbb{R})}^{2}
$$

for $\int \dot{U} \partial \underline{U}=0$, thanks to the following classical observation.

LEMMA 4 For a Sturm-Liouville operator $\mathcal{M}=-\partial K \partial+\alpha$ with $\alpha$ bounded and $K \geqslant K_{0}>0$, it is possible to equip $H^{1}$ with a modified inner product, whose norm is equivalent to the usual one, such that if $\mathcal{M} \cdot V=0$ then the orthogonal of $V$ in $L^{2}(\mathbb{R})$ coincides with the orthogonal of $V$ in $H^{1}(\mathbb{R})$.

Proof. For $\lambda>0$ large enough

$$
\langle U, V\rangle=\int(K \partial U \partial V+(\alpha+\lambda) U V)
$$


defines an inner product on $H^{1}$ as required. If $\mathcal{M} \cdot V=0$ then

$$
\langle U, V\rangle=\int U(\mathcal{M}+\lambda) \cdot V=\lambda \int V U,
$$

hence $\int V U=0$ is equivalent to $\langle U, V\rangle=0$.

REMARK 3 For a monotone $\underline{\mathbf{U}}$, the fact that the spectrum of the linearized operator $L$ is purely imaginary may be viewed as a consequence of the monotonicity of $\mathcal{L}$. This follows from Lemma 3.1 in [31]. Another consequence of this general result is an upper bound for the number of unstable eigenvalues for the linearized operator $L$ about solitons (which are not monotone). More precisely, if $\mathbf{U}$ is a soliton in our context, $\partial U_{1}$ has a single zero and this implies by Sturm-Liuouville theory (see Appendix B) that $\mathcal{L}$ has a single negative eigenvalue. Hence by Lemma 3.1 in [31] the linearized operator $L$ about $\underline{\mathbf{U}}$ has at most one unstable eigenvalue.

The approach developed in [18, 9] aims at selecting orbitally stable solitons, for which $L$ has no unstable eigenvalue. We adapt below that approach to solitons with non-zero endstates.

From now on, to simplify the writing, we denote by $v=U_{1}$ and $u=U_{2}$ the components of any vector $\mathbf{U}$ in the state space 11 Let us consider a soliton $\underline{\mathbf{U}}=(\underline{v}, \underline{u})$ with endstate $\mathbf{U}_{\infty}=\left(v_{\infty}, u_{\infty}\right)$ and speed $\sigma$. It satisfies the ODE

$$
\delta \mathcal{H}[\underline{\mathbf{U}}]+\sigma \mathbf{J}_{\varepsilon} \underline{\mathbf{U}} \equiv \delta \mathcal{H}\left[\mathbf{U}_{\infty}\right]+\sigma \mathbf{J}_{\varepsilon} \mathbf{U}_{\infty}
$$

with

$$
\mathbf{J}_{\varepsilon}:=\varepsilon\left(\begin{array}{ll}
0 & 1 \\
1 & 0
\end{array}\right), \quad \varepsilon \in\{-1,1\} .
$$

(Recall that $\varepsilon=-1$ when we consider the Eulerian model (1.4) and that $\varepsilon=1$ for the Lagrangian one (1.5.) We define here

$$
\begin{array}{rlrl}
\mathcal{H}\left[\underline{\mathbf{U}} ; \mathbf{U}_{\infty}\right] & =\int_{-\infty}^{\infty}\left(H[\underline{\mathbf{U}}]-H\left[\mathbf{U}_{\infty}\right]\right)(\xi) \mathrm{d} \xi, & \mathcal{Q}\left[\underline{\mathbf{U}} ; \mathbf{U}_{\infty}\right]=\int_{-\infty}^{\infty}\left(\underline{v u}-v_{\infty} u_{\infty}\right)(\xi) \mathrm{d} \xi, \\
\mathcal{P}_{1}\left[\underline{\mathbf{U}} ; \mathbf{U}_{\infty}\right]=\int_{-\infty}^{\infty}\left(\underline{\nu}-v_{\infty}\right)(\xi) \mathrm{d} \xi, & \mathcal{P}_{2}\left[\underline{\mathbf{U}} ; \mathbf{U}_{\infty}\right]=\int_{-\infty}^{\infty}\left(\underline{u}-u_{\infty}\right)(\xi) \mathrm{d} \xi,
\end{array}
$$

where all the integrals are absolutely convergent since $\underline{\mathbf{U}}$ converges exponentially fast to $\mathbf{U}_{\infty}$ at $\pm \infty$ (recall that $\underline{\mathbf{U}}$ is a saddle-saddle connection). Furthermore, denoting by $\lambda_{i}\left(\sigma ; \mathbf{U}_{\infty}\right)$ the components of $\delta \mathcal{H}\left[\mathbf{U}_{\infty}\right]+\sigma \mathbf{J}_{\varepsilon} \mathbf{U}_{\infty}, i=1,2$, we see that equation (3.17) equivalently reads

$$
\left(\delta \mathcal{H}+\varepsilon \sigma \delta \mathcal{Q}-\lambda_{1}\left(\sigma ; \mathbf{U}_{\infty}\right) \delta \mathcal{P}_{1}-\lambda_{2}\left(\sigma ; \mathbf{U}_{\infty}\right) \delta \mathcal{P}_{2}\right)\left[\underline{\mathbf{U}} ; \mathbf{U}_{\infty}\right] \equiv 0,
$$

the variational gradient being taken with respect to $\underline{\mathbf{U}}$ only. This implies in particular that

$$
\left(\mathcal{H}+\varepsilon \sigma \mathcal{Q}-\lambda_{1}\left(\sigma ; \mathbf{U}_{\infty}\right) \mathcal{P}_{1}-\lambda_{2}\left(\sigma ; \mathbf{U}_{\infty}\right) \mathcal{P}_{2}\right)\left[\underline{\mathbf{U}} ; \mathbf{U}_{\infty}\right]
$$

is invariant under shifting the profile $\underline{\mathbf{U}}$ into $\underline{\mathbf{U}}_{s}$. In other words, that quantity depends only on $\left(\sigma ; \mathbf{U}_{\infty}\right)$. Furthermore, this is an extension to non-zero endstates of what Bona and Sachs, after Boussinesq, call the moment of instability of the soliton $\underline{\mathbf{U}}$.

${ }^{1}$ So that in the Lagrangian framework, $v=v$, and in the Eulerian framework, $v=\rho$. 
DEFINITION 1 We define the moment of instability of a soliton $\underline{\mathbf{U}}$ with endstate $\mathbf{U}_{\infty}$ and speed $\sigma$ to be the quantity

$$
m\left(\sigma ; \mathbf{U}_{\infty}\right):=\left(\mathcal{H}+\varepsilon \sigma \mathcal{Q}-\lambda_{1}\left(\sigma ; \mathbf{U}_{\infty}\right) \mathcal{P}_{1}-\lambda_{2}\left(\sigma ; \mathbf{U}_{\infty}\right) \mathcal{P}_{2}\right)\left[\underline{\mathbf{U}} ; \mathbf{U}_{\infty}\right]
$$

As we shall see in Section 3.3 it is the local convexity of $m$ as a function of $\sigma$ which determines the stability of the soliton $\underline{\mathbf{U}}$. It is interesting to note that the evaluation of $m$ does not require the actual resolution of the ODE 3.17).

We begin with the Lagrangian model, for which the computations are simpler.

Proposition 3 The moment of instability of a soliton $\underline{\mathbf{U}}=(\underline{v}, \underline{u})$ of $(1.5)$ is

$$
m=\int_{-\infty}^{+\infty} \kappa(\underline{v}(\zeta))(\underline{\dot{v}}(\zeta))^{2} \mathrm{~d} \zeta
$$

Alternatively, if $\underline{v}$ achieves a maximum $v_{M}>v_{\infty}$, then

$$
m=2 \int_{v_{\infty}}^{v_{M}} \sqrt{2 \kappa(v)\left(f_{0}(v)-f_{0}\left(v_{\infty}\right)+p_{0}\left(v_{\infty}\right)\left(v-v_{\infty}\right)-\frac{1}{2} \tau^{2}\left(v-v_{ \pm}\right)^{2}\right)} \mathrm{d} v
$$

while

$$
m=2 \int_{v_{m}}^{v_{\infty}} \sqrt{2 \kappa(v)\left(f_{0}(v)-f_{0}\left(v_{\infty}\right)+p_{0}\left(v_{\infty}\right)\left(v-v_{\infty}\right)-\frac{1}{2} \tau^{2}\left(v-v_{ \pm}\right)^{2}\right)} \mathrm{d} v
$$

if $\underline{v}$ achieves a minimum $v_{m}<v_{\infty}$.

Proof. The formula in [3.18) is an extension of the one used in [9. p. 26] for a power law for $p_{0}$ and zero endstate. It follows from the elimination of the speed

$$
\underline{u}=u_{\infty}+\tau\left(v_{\infty}-\underline{v}\right)
$$

in the definition of $m$ and the use of the identity

$$
\frac{1}{2} \kappa\left(\underline{v} \underline{\dot{v}}^{2}+f_{0}\left(v_{\infty}\right)-f_{0}(\underline{v})-p_{0}\left(v_{\infty}\right)\left(\underline{v}-v_{\infty}\right)+\frac{1}{2} \tau^{2}\left(\underline{v}-v_{\infty}\right)^{2} \equiv 0,\right.
$$

where $\tau$ denotes the "speed" of the soliton (we use $\tau$ instead of $\sigma$ here for consistency with the notation used in Section 2). As a matter of fact, by definition

$$
\lambda_{1}=-p_{0}\left(v_{\infty}\right)+\tau u_{\infty}, \quad \lambda_{2}=u_{\infty}+\tau v_{\infty},
$$

hence

$$
\begin{aligned}
H[\underline{\mathbf{U}}]-H\left[\mathbf{U}_{\infty}\right]+\tau\left(\underline{v} \underline{u}-v_{\infty} u_{\infty}\right)-\lambda_{1}\left(\underline{v}-v_{\infty}\right)-\lambda_{2}\left(\underline{u}-u_{\infty}\right) \\
\\
=\kappa(\underline{v}) \underline{\dot{v}}^{2}+\frac{1}{2}\left(\underline{u}-u_{\infty}+\tau\left(\underline{v}-v_{\infty}\right)\right)^{2}=\kappa(\underline{v}) \underline{\dot{v}}^{2} .
\end{aligned}
$$

The other representation of $m$ merely follows from 3.21 and the change of variables $v=$ $\underline{v}(\zeta)$, which is valid on the two half-lines separated by the point where $\underline{v}$ achieves its (unique) extremum. 
REMARK 4 Physically, the quantity $m=\int \kappa(v) \underline{v}^{2}$ may be interpreted as (a non-equilibrium analogue of) surface tension [24].

REMARK 5 The advantage of the formulas in (3.19) and (3.20) is that they can easily be evaluated numerically, without solving the profile equation (3.17), the points $v_{m, M}$ just being characterized by the vanishing of the integrand. More precisely, $v_{M}$ is the smallest $v>v_{\infty}$ where

$$
f_{0}(v)-f_{0}\left(v_{\infty}\right)+p_{0}\left(v_{\infty}\right)\left(v-v_{\infty}\right)-\frac{1}{2} \tau^{2}\left(v-v_{ \pm}\right)^{2}=0,
$$

while $v_{m}$ is the greatest $v<v_{\infty}$ canceling the same expression. The existence/relevance of such points follows from the phase portrait analysis of Section 2

REMARK 6 The equivalent expressions (3.18) and (3.19) (or (3.20) of $m$ arise as soon as the Hamiltonian $\mathcal{H}$ satisfies 3.16 with $\mathcal{H}_{0}$ depending only on $\mathbf{U}$ (and not on the derivatives of $\mathbf{U}$ ). Indeed, with the notation introduced in Section 3.3.a below, the soliton equation (3.17) equivalently reads

and the counterpart of 3.16 regarding $\widetilde{\mathcal{H}}$ reads

$$
\delta \tilde{\mathcal{H}}[\underline{\tilde{\mathbf{U}}}]+\varepsilon \sigma \delta \widetilde{\mathcal{Q}}[\underline{\tilde{\mathbf{U}}}] \equiv 0,
$$

$$
\tilde{\mathcal{H}}[\underline{\tilde{\mathbf{U}}}]=\tilde{\mathcal{H}}_{0}[\underline{\tilde{\mathbf{U}}}]+\mathcal{K}\left[\underline{U}_{1}\right] .
$$

We infer that $\partial \underline{\mathbf{U}}$ is an integrating factor of the soliton equation and that

$$
\widetilde{H}_{0}(\underline{\tilde{\mathbf{U}}})+\varepsilon \sigma \widetilde{Q}(\underline{\tilde{\mathbf{U}}})-\frac{1}{2} k\left(\underline{U}_{1}\right)\left(\partial \underline{U}_{1}\right)^{2} \equiv 0,
$$

with $\widetilde{H}_{0}(\underline{\widetilde{\mathbf{U}}})$ and $\widetilde{Q}(\underline{\widetilde{\mathbf{U}}})$ being naturally defined as the integrands in the functionals $\widetilde{H}_{0}[\underline{\widetilde{\mathbf{U}}}]$ and $\widetilde{Q}[\underline{\widetilde{\mathbf{U}}}]$ respectively. Therefore

$$
\widetilde{\mathcal{H}}[\underline{\widetilde{\mathbf{U}}}]+\varepsilon \sigma \widetilde{\mathcal{Q}}[\underline{\widetilde{\mathbf{U}}}]=2 \mathcal{K}\left[\underline{U}_{1}\right],
$$

and the left-hand side is exactly $m\left(\sigma ; \mathbf{U}_{\infty}\right)$ (see Section 3.3.a below).

\subsection{Orbital stability of capillary profiles}

The long time analysis of a model of course necessitates some knowledge on its local wellposedness. As regards the Korteweg models (1.4) and (1.5), this question has been addressed in [7]. Recall in particular the following.

THEOREM 1 For any global smooth solution $\widehat{\mathbf{U}}$ of $(1.5)$, for all $\mathbf{U}_{0}=\left(v_{0}, u_{0}\right) \in \widehat{\mathbf{U}}+\left(H^{3} \times H^{2}\right)(\mathbb{R})$ such that $v_{0}$ takes values in a compact subset of $(b,+\infty)$, there exists $T>0$ and a unique solution $\mathbf{U}$ of (1.5) such that $\left.\mathbf{U}\right|_{t=0}=\mathbf{U}_{0}$ and

$$
\mathbf{U}-\widehat{\mathbf{U}} \in \mathcal{C}\left([0, T] ;\left(H^{3} \times H^{2}\right)(\mathbb{R})\right) \cap \mathcal{C}^{1}\left([0, T] ;\left(H^{1} \times L^{2}\right)(\mathbb{R})\right) .
$$

If $\widehat{\mathbf{U}}$ is a traveling wave with profile $\underline{\mathbf{U}}$, the Sobolev norms of $\partial_{y} \widehat{\mathbf{U}}$ do not depend on $t$ and it can be shown that the time $T$ of existence satisfies a lower bound

$$
T \geqslant C \ln \left(1+\frac{1}{\left\|u_{0}-\underline{u}\right\|_{H^{2}}+\left\|\partial_{y}\left(v_{0}-\underline{v}\right)\right\|_{H^{2}}}\right) .
$$

In the semi-linear case $\kappa \equiv$ constant, a much stronger result is known, which says that an a priori bound in $\left(H^{1} \times L^{2}\right)(\mathbb{R})$ implies that the maximal solution is global (see [9, Theorem 4, p. 20]). 
DEFINITION 2 We say that a traveling wave $\widehat{\mathbf{U}}$ with profile $\underline{\mathbf{U}}$ is an orbitally stable solution of (3.15) if there exists $\varepsilon_{1}>0$ such that for all $\varepsilon \in\left(0, \varepsilon_{1}\right]$, there exists $\eta>0$ such that for any $\mathbf{U} \in \underline{\mathbf{U}}+\mathcal{C}\left([0, T) ; H^{3} \times H^{1}\right)$ which is a solution of [3.15],

$$
\|\mathbf{U}(0)-\underline{\mathbf{U}}\|_{H^{1} \times L^{2}}<\eta \quad \text { implies } \quad \inf _{s \in \mathbb{R}}\left\|\mathbf{U}(t)-\underline{\mathbf{U}}_{s}\right\|_{H^{1} \times L^{2}}<\varepsilon
$$

for all $t \in[0, T)$.

REMARK 7 If $\underline{\mathbf{U}}$ is a soliton, then $\left\|\underline{\mathbf{U}}-\underline{\mathbf{U}}_{s}\right\|_{H^{1} \times L^{2}}$ is uniformly bounded in $s$. Therefore, the orbital stability of a soliton implies an a priori bound for $\|\mathbf{U}(t)-\underline{\mathbf{U}}\|_{H^{1} \times L^{2}}$. As mentioned above, this is sufficient to have global existence in the special case $\kappa \equiv$ constant in 1.5 but not in general.

\section{3.a Solitons}

THEOREM 2 A solitary wave solution $\widehat{\mathbf{U}}$ of 3.15 with endstate $\mathbf{U}_{\infty}$ and speed $\sigma$ is orbitally stable if its moment of instability (Definition 1) satisfies

$$
\frac{\partial^{2} m}{\partial \sigma^{2}}\left(\sigma ; \mathbf{U}_{\infty}\right)>0
$$

Proof. It is a direct modification of Theorem 5 in [9], which is itself an application of Theorem 2 in [18]. Indeed, we can force our model to enter the framework of [18] by: 1) shifting the dependent variables $\mathbf{U}$ by $\mathbf{U}_{\infty}$ so that in the new variables the soliton becomes homoclinic to 0 and 2) modifying the Hamiltonian to make its gradient vanish at infinity. More precisely, setting

$$
\tilde{\mathbf{U}}:=\mathbf{U}-\mathbf{U}_{\infty} \quad \text { and } \quad \tilde{\mathcal{H}}[\tilde{\mathbf{U}}]:=\mathcal{H}\left[\mathbf{U} ; \mathbf{U}_{\infty}\right]-\int \delta \mathcal{H}\left[\mathbf{U}_{\infty}\right] \cdot\left(\mathbf{U}-\mathbf{U}_{\infty}\right)
$$

we have

$$
\delta \tilde{\mathcal{H}}[\tilde{\mathbf{U}}]=\delta \mathcal{H}[\mathbf{U}]-\delta \mathcal{H}\left[\mathbf{U}_{\infty}\right]
$$

and the abstract equation 3.15 equivalently reads

$$
\partial_{t} \widetilde{\mathbf{U}}=\mathcal{J} \delta \tilde{\mathcal{H}}[\tilde{\mathbf{U}}] .
$$

This is because $\mathcal{J}$ vanishes at constants. Furthermore, $\underline{\widetilde{\mathbf{U}}}:=\underline{\mathbf{U}}-\mathbf{U}_{\infty}$ is a critical point of $\tilde{\mathcal{H}}$ under the single constraint

$$
\widetilde{\mathcal{Q}}[\widetilde{\mathbf{U}}]:=\int \widetilde{v} \widetilde{u}
$$

in that it satisfies the ODE

$$
\delta \widetilde{\mathcal{H}}[\underline{\tilde{\mathbf{U}}}]+\varepsilon \sigma \delta \widetilde{\mathcal{Q}}[\underline{\tilde{\mathbf{U}}}] \equiv 0 .
$$

Finally, our definition of

$$
m\left(\sigma ; \mathbf{U}_{\infty}\right)=\tilde{\mathcal{H}}[\tilde{\mathbf{U}}]+\varepsilon \sigma\left(\mathcal{Q}-u_{\infty} \mathcal{P}_{1}-v_{\infty} \mathcal{P}_{2}\right)\left[\underline{\mathbf{U}}, \mathbf{U}_{\infty}\right]=\tilde{\mathcal{H}}[\widetilde{\mathbf{U}}]+\varepsilon \sigma \widetilde{\mathcal{Q}}[\widetilde{\mathbf{U}}]
$$

coincides with the moment of instability of [9, 18] for the soliton $\underline{\widetilde{\mathbf{U}}}$ of 3.23 . 
REMARK 8 Theorem 2 in [18] is actually an "if and only if" result. But the "only if" part requires that the operator $\mathcal{J}$ be onto 1 which is obviously not the case here (nor in [9]). This problem is fixed in [10] for Korteweg-de Vries type equations. Up to our knowledge, there is no equivalent work on the Boussinesq equation.

To help the reader understand the significance of $m$ we recall from [10, 18] an important result concerning instability. For a clear statement, we use a notation in which the dependence on the wave speed $\sigma$ is more explicit. We choose to add $\sigma$ as a superscript, in such a way that $\underline{\mathbf{U}}^{\sigma}$ stands for a traveling profile with speed $\sigma$. We omit the dependence on $\mathbf{U}_{\infty}$, which is fixed in what follows. For consistency of notation, the moment of instability is now denoted $m^{\sigma}$.

Proposition 4 If the moment of instability of a soliton $\underline{\mathbf{U}}^{\tau}$ satisfies

$$
\left.\frac{\partial^{2} m^{\sigma}}{\partial \sigma^{2}}\right|_{\sigma=\tau} \leqslant 0
$$

there exists a smooth curve $\sigma \mapsto \mathbf{V}^{\sigma} \in \mathbf{U}_{\infty}+\mathcal{D}(\mathcal{L})$ (the domain of $\mathcal{L}$ ) such that $\mathbf{V}^{\tau}=\underline{\mathbf{U}}^{\tau}$ and for all $\sigma$ close to $\tau$,

$$
\widetilde{\mathcal{Q}}\left[\mathbf{V}^{\sigma}-\mathbf{U}_{\infty}\right]=\widetilde{\mathcal{Q}}\left[\underline{\mathbf{U}}^{\tau}-\mathbf{U}_{\infty}\right] \quad \text { and } \quad \tilde{\mathcal{H}}\left[\mathbf{V}^{\sigma}-\mathbf{U}_{\infty}\right]<\tilde{\mathcal{H}}\left[\underline{\mathbf{U}}^{\tau}-\mathbf{U}_{\infty}\right]
$$

for $\sigma \neq \tau$.

Proof. We follow the second proof of Theorem 3.1 in [10] (see also Theorem 4.1 in [18], for a different parametrization). We first observe that

$$
\begin{gathered}
\frac{\partial m^{\sigma}}{\partial \sigma}=\varepsilon \int\left(\underline{v}^{\sigma}-v_{\infty}\right)\left(\underline{u}^{\sigma}-u_{\infty}\right), \\
\frac{\partial^{2} m^{\sigma}}{\partial \sigma^{2}}=\varepsilon \int\left(\left(\underline{u}^{\sigma}-u_{\infty}\right) \frac{\partial \underline{v}^{\sigma}}{\partial \sigma}+\left(\underline{v}^{\sigma}-v_{\infty}\right) \frac{\partial \underline{u}^{\sigma}}{\partial \sigma}\right) .
\end{gathered}
$$

As a matter of fact, by definition

$$
m^{\sigma}=(\widetilde{\mathcal{H}}+\varepsilon \sigma \widetilde{\mathcal{Q}})\left[\underline{\mathbf{U}}^{\sigma}-\mathbf{U}_{\infty}\right]
$$

and the soliton equation is

$$
(\delta \tilde{\mathcal{H}}+\varepsilon \sigma \delta \widetilde{\mathcal{Q}})\left[\underline{\mathbf{U}}^{\sigma}-\mathbf{U}_{\infty}\right]=0 .
$$

Therefore

which is (3.24), and

$$
\varepsilon \frac{\partial m^{\sigma}}{\partial \sigma}=\widetilde{\mathcal{Q}}\left[\underline{\mathbf{U}}^{\sigma}-\mathbf{U}_{\infty}\right],
$$

$$
\varepsilon \frac{\partial^{2} m^{\sigma}}{\partial \sigma^{2}}=\int \delta \widetilde{\mathcal{Q}}\left[\underline{\mathbf{U}}^{\sigma}-\mathbf{U}_{\infty}\right] \cdot \frac{\partial \underline{\mathbf{U}}^{\sigma}}{\partial \sigma},
$$

which is 3.25). Additionally, by differentiation of 3.26), we get

$$
\mathcal{L}^{\sigma} \cdot \frac{\partial \underline{\mathbf{U}}^{\sigma}}{\partial \sigma}+\mathbf{J}_{\varepsilon} \cdot\left(\underline{\mathbf{U}}^{\sigma}-\mathbf{U}_{\infty}\right)=0,
$$

\footnotetext{
1 In fact, it requires only that $\underline{\mathbf{U}}^{\sigma}$ and $\mathbf{W}^{\sigma}$ (an eigenvector of $\mathcal{L}^{\sigma}$ defined in the proof of Proposition 4 belong to the range of $\mathcal{J}$ : this is not the case for $\underline{\mathbf{U}}^{\sigma}$, whose integral on $\mathbb{R}$ is non-zero.
} 
where the operator

$$
\mathcal{L}^{\sigma}:=\operatorname{Hess}(\tilde{\mathcal{H}}+\varepsilon \sigma \widetilde{\mathcal{Q}})\left[\underline{\mathbf{U}}^{\sigma}-\mathbf{U}_{\infty}\right]=\operatorname{Hess}(\mathcal{H}+\varepsilon \sigma \mathcal{Q})\left[\underline{\mathbf{U}}^{\sigma}\right]
$$

enjoys the same definition as in Lemma 3 . Note indeed that

$$
\begin{gathered}
\text { Hess } \tilde{\mathcal{H}}\left[\mathbf{U}-\mathbf{U}_{\infty}\right]=\operatorname{Hess} \mathcal{H}[\mathbf{U}], \quad \text { Hess } \widetilde{\mathcal{Q}}\left[\mathbf{U}-\mathbf{U}_{\infty}\right]=\varepsilon \mathbf{J}_{\varepsilon}, \\
\mathbf{J}_{\varepsilon} \cdot\left(\mathbf{U}-\mathbf{U}_{\infty}\right)=\varepsilon \delta \widetilde{\mathcal{Q}}\left[\mathbf{U}-\mathbf{U}_{\infty}\right] .
\end{gathered}
$$

Thanks to 3.27) we see that 3.25 equivalently reads

$$
\frac{\partial^{2} m^{\sigma}}{\partial \sigma^{2}}=-\int\left(\frac{\partial \underline{\mathbf{U}}^{\sigma}}{\partial \sigma}\right)^{*} \mathcal{L}^{\sigma} \cdot \frac{\partial \underline{\mathbf{U}}^{\sigma}}{\partial \sigma} .
$$

This formula is valid independently of the convexity or concavity of $m$. It shows in particular that when $m$ is strictly convex (a stable case, according to Theorem 2), the operator $\mathcal{L}^{\sigma}$ does have a negative space. In fact, this is always the case: unlike the situation considered in Lemma 3 , here the profile $\underline{\mathbf{U}}^{\sigma}$ is not monotone. As already mentioned, this means that $\mathcal{L}^{\sigma}$ admits a negative eigenvalue. A more detailed analysis using the theory of Sturm-Liouville operators, postponed to Appendix B, shows that $\mathcal{L}^{\sigma}$ admits a single eigenvalue of negative real part, which is simple and associated with an eigenfunction, say $\mathbf{W}^{\sigma}$, of which the first component $\omega^{\sigma}$ does not vanish and the second component $w^{\sigma}$ has a simple expression in terms of $\omega^{\sigma}, \sigma$ (and $\underline{\mathbf{U}}^{\sigma}$ in the Eulerian framework): in the Lagrangian framework for instance, we have

$$
(\lambda-1) w^{\sigma}=\sigma \omega^{\sigma}
$$

if $\lambda$ denotes the negative eigenvalue, hence in particular $\sigma \omega^{\sigma} w^{\sigma} \leqslant 0$ (regarding dimensional questions, see Remark 13 in Appendix B). Now we look for

$$
\mathbf{V}^{\sigma}=\underline{\mathbf{U}}^{\sigma}+\varphi(\sigma) \mathbf{W}^{\tau}
$$

with $\varphi$ a smooth function vanishing at $\sigma=\tau$. We have

$$
\begin{aligned}
\left.\frac{\partial}{\partial \varphi} \widetilde{\mathcal{Q}}\left[\underline{\mathbf{U}}^{\sigma}+\varphi \mathbf{W}^{\tau}-\mathbf{U}_{\infty}\right]\right|_{(\sigma, \varphi)=(\tau, 0)} & =\delta \widetilde{\mathcal{Q}}\left[\underline{\mathbf{U}}^{\tau}-\mathbf{U}_{\infty}\right] \cdot \mathbf{W}^{\tau} \\
& =\int\left(\left(\underline{v}^{\tau}-v_{\infty}\right) \omega^{\tau}+\left(\underline{u}^{\tau}-u_{\infty}\right) w^{\tau}\right) .
\end{aligned}
$$

In the Lagrangian framework in particular, this integral reduces to

$$
\int\left(\underline{v}^{\tau}-v_{\infty}\right)\left(\omega^{\tau}-\tau w^{\tau}\right)
$$

and is therefore non-zero because of the properties $\tau \omega^{\tau} w^{\tau} \leqslant 0, \omega^{\tau} \neq 0$ and $\underline{v}^{\tau}-v_{\infty} \neq 0$. Similar arguments work in the Eulerian framework. Therefore, the existence of a function $\sigma \mapsto \varphi(\sigma)$ such that

$$
\widetilde{\mathcal{Q}}\left[\underline{\mathbf{U}}^{\sigma}+\varphi(\sigma) \mathbf{W}^{\tau}-\mathbf{U}_{\infty}\right]=\widetilde{\mathcal{Q}}\left[\underline{\mathbf{U}}^{\tau}-\mathbf{U}_{\infty}\right]
$$

follows from the implicit function theorem. Hence, $\mathbf{V}^{\sigma}=\underline{\mathbf{U}}^{\sigma}+\varphi(\sigma) \mathbf{W}^{\tau}$ is such that

$$
\tilde{\mathcal{H}}\left[\mathbf{V}^{\sigma}-\mathbf{U}_{\infty}\right]+\varepsilon \sigma \widetilde{\mathcal{Q}}\left[\mathbf{V}^{\sigma}-\mathbf{U}_{\infty}\right]=\tilde{\mathcal{H}}\left[\mathbf{V}^{\sigma}-\mathbf{U}_{\infty}\right]+\varepsilon \sigma \widetilde{\mathcal{Q}}\left[\underline{\mathbf{U}}^{\tau}-\mathbf{U}_{\infty}\right]
$$


Differentiating once with respect to $\sigma$ and evaluating at $\sigma=\tau$ we get, using 3.26 ,

$$
0=\left.\frac{\partial}{\partial \sigma} \tilde{\mathcal{H}}\left[\mathbf{V}^{\sigma}-\mathbf{U}_{\infty}\right]\right|_{\sigma=\tau} .
$$

Differentiating twice we obtain

$$
\int\left(\frac{\partial \mathbf{V}^{\sigma}}{\partial \sigma}\right)^{*} \mathcal{L}^{\sigma} \cdot \frac{\partial \mathbf{V}^{\sigma}}{\partial \sigma}=\frac{\partial^{2}}{\partial \sigma^{2}} \tilde{\mathcal{H}}\left[\mathbf{V}^{\sigma}-\mathbf{U}_{\infty}\right]
$$

at $\sigma=\tau$, with

$$
\frac{\partial \mathbf{V}^{\sigma}}{\partial \sigma}=\frac{\partial \underline{\mathbf{U}}^{\sigma}}{\partial \sigma}+\frac{\mathrm{d} \varphi}{\mathrm{d} \sigma} \mathbf{W}^{\tau}
$$

and

$$
0=\int \delta \widetilde{Q}\left[\mathbf{V}^{\sigma}-\mathbf{U}_{\infty}\right] \cdot \frac{\partial \mathbf{V}^{\sigma}}{\partial \sigma}=\int\left(\frac{\partial \mathbf{V}^{\sigma}}{\partial \sigma}\right)^{*} \mathbf{J}_{\varepsilon} \cdot\left(\mathbf{V}^{\sigma}-\mathbf{U}_{\infty}\right)
$$

which implies

$$
\frac{\mathrm{d} \varphi}{\mathrm{d} \sigma} \int\left(\mathbf{W}^{\tau}\right)^{*} \mathbf{J}_{\varepsilon} \cdot\left(\underline{\mathbf{U}}^{\tau}-\mathbf{U}_{\infty}\right)+\int\left(\underline{\mathbf{U}}^{\tau}-\mathbf{U}_{\infty}\right)^{*} \mathbf{J}_{\varepsilon} \cdot \frac{\partial \underline{\mathbf{U}}^{\sigma}}{\partial \sigma}=0
$$

at $\sigma=\tau$. We thus have, using 3.27,

$$
\begin{aligned}
\frac{\partial^{2}}{\partial \sigma^{2}} \widetilde{\mathcal{H}}\left[\mathbf{V}^{\sigma}-\mathbf{U}_{\infty}\right]=-\varepsilon \int\left(\frac{\partial \mathbf{V}^{\sigma}}{\partial \sigma}\right)^{*} \mathbf{J}_{\varepsilon} \cdot\left(\underline{\mathbf{U}}^{\tau}-\mathbf{U}_{\infty}\right)+\frac{\mathrm{d} \varphi}{\mathrm{d} \sigma} \int\left(\frac{\partial \mathbf{V}^{\sigma}}{\partial \sigma}\right)^{*} \mathcal{L}^{\tau} \cdot \mathbf{W}^{\tau} \\
=\frac{\mathrm{d} \varphi}{\mathrm{d} \sigma} \int\left(\mathbf{W}^{\tau}\right)^{*} \mathcal{L}^{\tau} \cdot \frac{\partial \mathbf{V}^{\sigma}}{\partial \sigma}=-\varepsilon \frac{\mathrm{d} \varphi}{\mathrm{d} \sigma} \int\left(\mathbf{W}^{\tau}\right)^{*} \mathbf{J}_{\varepsilon} \cdot\left(\underline{\mathbf{U}}^{\tau}-\mathbf{U}_{\infty}\right)+\left(\frac{\mathrm{d} \varphi}{\mathrm{d} \sigma}\right)^{2} \int\left(\mathbf{W}^{\tau}\right)^{*} \mathcal{L}^{\tau} \cdot \mathbf{W}^{\tau} \\
\quad=\varepsilon \int\left(\underline{\mathbf{U}}^{\tau}-\mathbf{U}_{\infty}\right)^{*} \mathbf{J}_{\varepsilon} \cdot \frac{\partial \underline{\mathbf{U}}^{\sigma}}{\partial \sigma}+\left(\frac{\mathrm{d} \varphi}{\mathrm{d} \sigma}\right)^{2} \int\left(\mathbf{W}^{\tau}\right)^{*} \mathcal{L}^{\tau} \cdot \mathbf{W}^{\tau}
\end{aligned}
$$

at $\sigma=\tau$, where we have used the self-adjointness of $\mathcal{L}^{\tau}$ (in $L^{2}(\mathbb{R})$ ) and $\mathbf{J}_{\varepsilon}$ (in $\mathbb{R}^{2}$ ). In the last right-hand side we recognize the second derivative of $m^{\sigma}$, which is non-positive by assumption. Since $\mathbf{W}^{\tau}$ is a negative eigenfunction of $\mathcal{L}^{\tau}, \int\left(\mathbf{W}^{\tau}\right)^{*} \mathcal{L}^{\tau} \cdot \mathbf{W}^{\tau}$ is negative and therefore the second derivative of $\widetilde{\mathcal{H}}$ is negative. This proves the proposition by Taylor expansion.

Proposition 4 means that, for points $\sigma$ where $m^{\sigma}$ is concave, $\widetilde{\mathcal{H}}$ is not locally minimized at $\underline{\mathbf{U}}^{\sigma}-\mathbf{U}_{\infty}$ under the constraint associated with $\widetilde{\mathcal{Q}}$. We suspect that this implies the instability of the profile but we have no proof. Additionally, the numerical plots of $m$ for the four types of solitons exhibited in Proposition 2 suggest that many of them are stable, depending on their "speed" (in fact, the mass transfer flux across the wave) and on the stability of the endstate. Recall that states inside the spinodal region, where $p_{0}$ increases with $v$, are considered unstable, both from the physical and from the mathematical point of view. To go further, it is important to have in mind the notion of metastable states.

DEFINITION 3 Under our main assumptions on $p_{0}$ and $f_{0}$, in the case when $p_{0}$ is increasing in some interval $\left(v_{*}, v^{*}\right) \subset(b,+\infty)$, the corresponding thermodynamical states are called spinodal. Moreover, the Maxwell or equilibrium states are uniquely defined by

$$
p_{0}\left(v_{m}\right)=p_{0}\left(v^{m}\right)=: p^{m}, \quad \int_{v_{m}}^{v^{m}}\left(p_{0}(v)-p^{m}\right) \mathrm{d} v=0, \quad v_{m}<v_{*}<v^{*}<v^{m} .
$$


The thermodynamical states with $v \in\left(v_{m}, v_{*}\right)$ or $v \in\left(v^{*}, v^{m}\right)$ are called metastable. States with $v \in\left(b, v_{m}\right)$ or $v \in\left(v^{m},+\infty\right)$ are called stable.

We provide and comment below several results that hopefully give some insight, even though they cannot be exhaustive. The graphs on the left of figures show the moment of instability $m$ as a function of $\tau$ (arbitrarily chosen positive; of course there are symmetric counterparts for negative $\tau$, which have the same convexity properties). The graphs on the right are intended to show Amplitude B of the solitons. They show, also as a function of $\tau$, either the maximum or the minimum of the soliton (depending on its type), which is obtained naturally in the computation of $m$ as the endpoint $v_{M}$ of the integral in 3.19) or $v_{m}$ in 3.20), characterized by $I\left(v_{M} ; v_{\infty}, \tau\right)=0$ or $I\left(v_{m} ; v_{\infty}, \tau\right)=0$. There are at least 50 computed points in all plots. The numerical values of Maxwell states with the pressure law we use (the van der Waals law for water at $T=600 \mathrm{~K}$ ) are

$$
v_{m}=58.7 \quad \text { and } \quad v^{m}=182.6
$$

REMARK 9 The pure vapor solitons considered in Figure 6 exist for $\tau^{2}$ in some finite interval $(0, S)$, their amplitude going to infinity (respectively 0 ) when $\tau$ tends to 0 (respectively $S$, which corresponds to the coincidence of the points denoted $v_{2}$ and $v_{3}$ before). In contrast to the usual feature of solitons in the water waves theory for instance, the "fastest" are the "smallest". We did not compute on the whole interval $(0, S)$ but on a rather large subinterval, on which the moment of instability $m$ looks convex. This might be confusing because of the large range of $m$. However, zooms on smaller intervals have shown no failure of convexity. This suggests that all pure vapor solitons are stable.
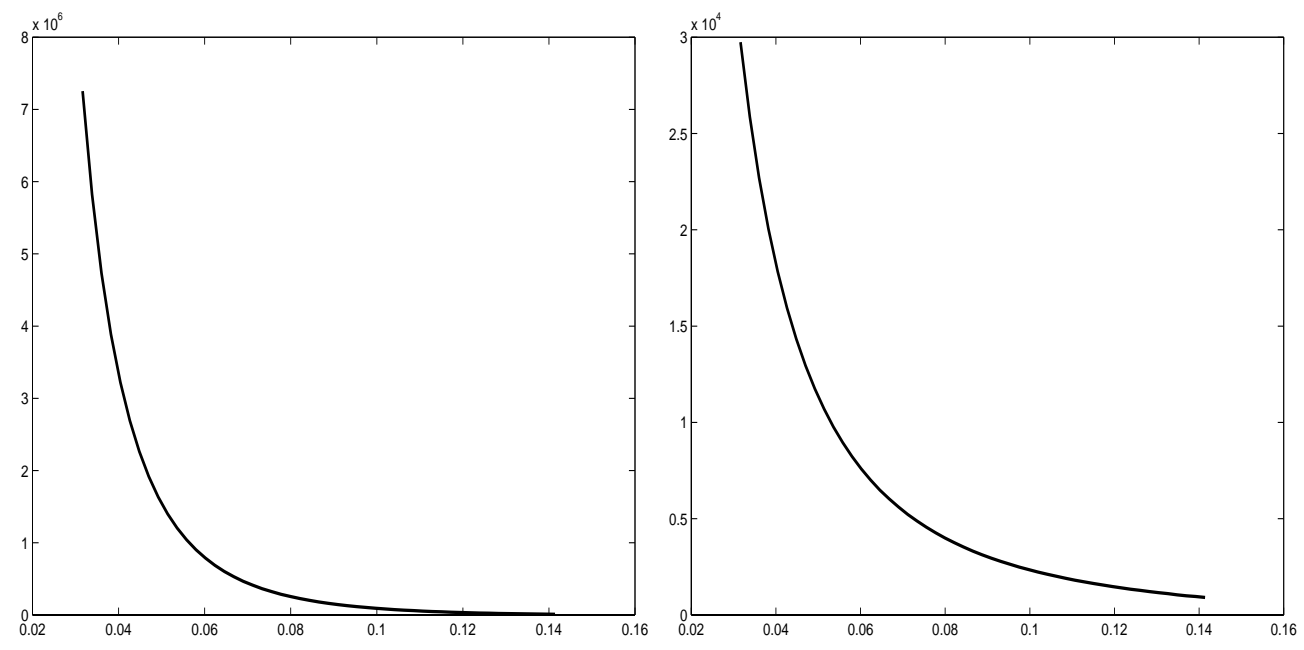

FIG. 6. Moment of instability (left) and maximum (right) of solitons with the endstate equal to the vapor saddle point of Figures 3 and $4\left(v_{\infty}=201.179\right)$.

REMARK 10 The temperate mixed liquid solitons considered in Figure 7, where the endstate is in the stable liquid phase, exist for $\tau^{2}$ in some finite interval $\left(S_{m}, S_{M}\right)$. When $\tau^{2}$ tends to the minimal value $S_{m}$, the maximum of the soliton tends to the vapor saddle point of the hereteroclinic connection 

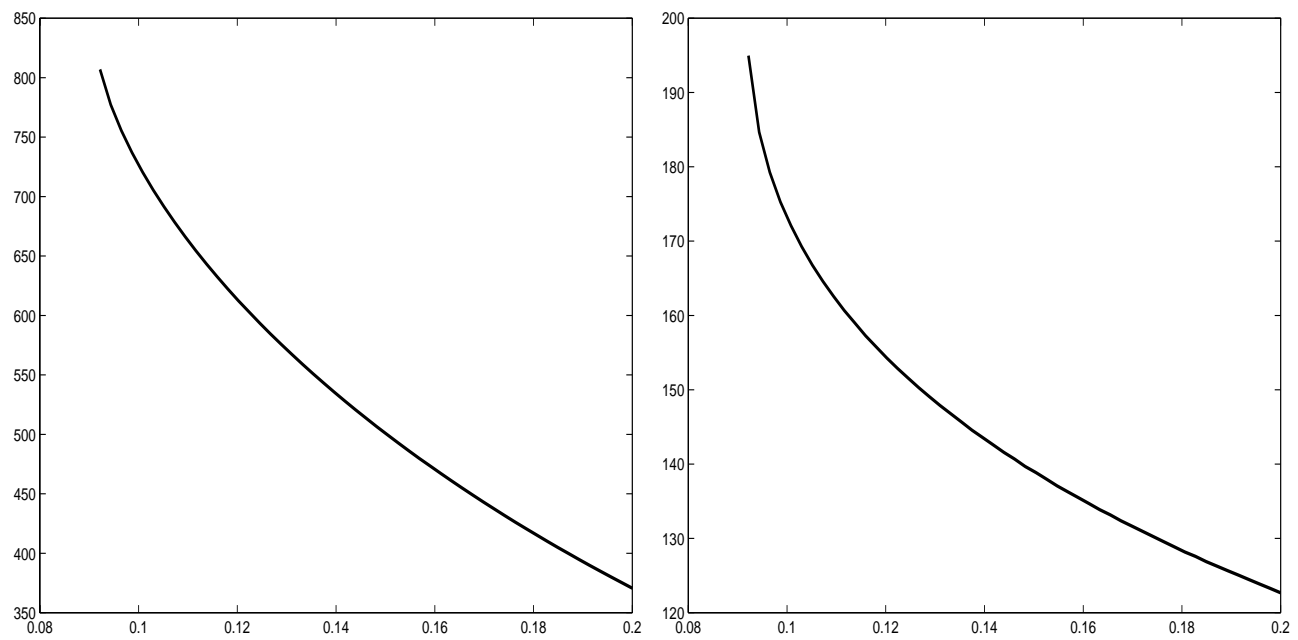

FIG. 7. Moment of instability (left) and maximum (right) of solitons with the endstate equal to the liquid saddle point of Figures 4 and $5\left(v_{\infty}=50.018\right)$
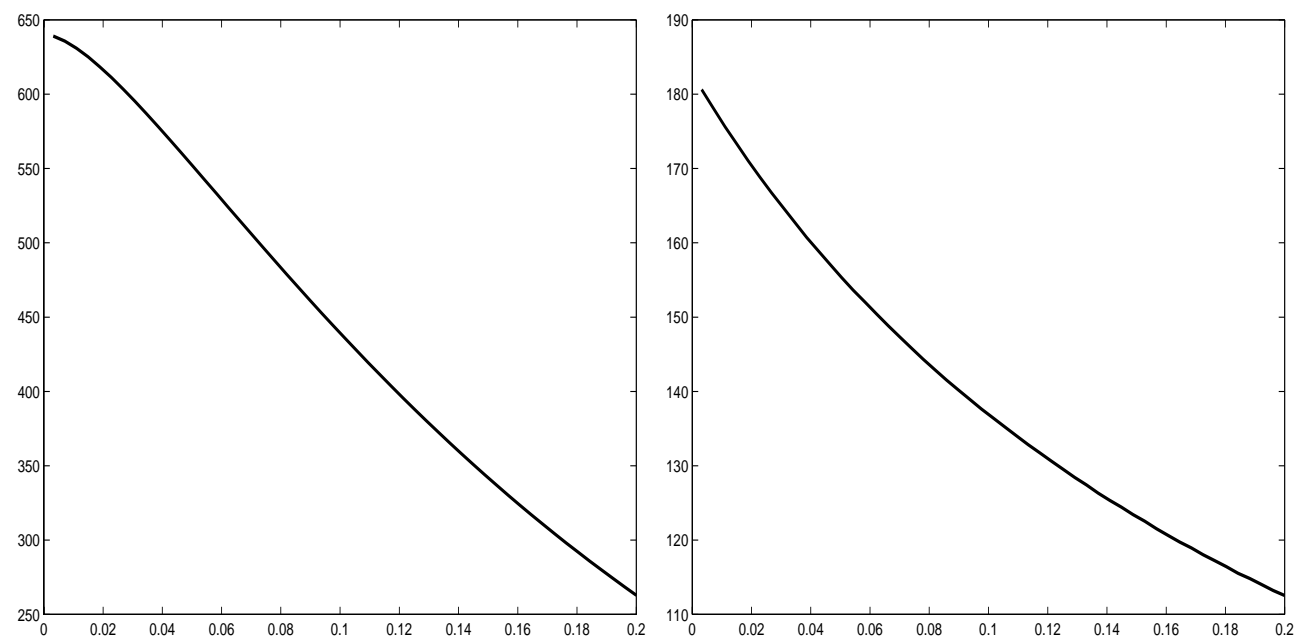

FIG. 8. Moment of instability (left) and maximum (right) of solitons with the endstate equal to the liquid Maxwell point $\left(v_{\infty}=58.678\right)$.

in Figure 4 (and the width of the soliton tends to infinity, as the orbit stays longer and longer in the neighborhood of the vapor saddle point). When $\tau^{2}$ tends to the maximal value $S_{M}$, the amplitude of the soliton tends to 0 (which corresponds to the coincidence of the points denoted $v_{0}$ and $v_{1}$ before). As far as we have approached the interval $\left(S_{m}, S_{M}\right)$, we have obtained a convex graph of the moment of instability $m$. Now, when the endstate is taken closer and closer to the Maxwell liquid point, the minimal value $S_{m}$ approaches 0 . Figure 8 shows that for the endstate equal to the Maxwell liquid point, the graph of the moment of instability $m$ exhibits a lack of convexity near 0 . 

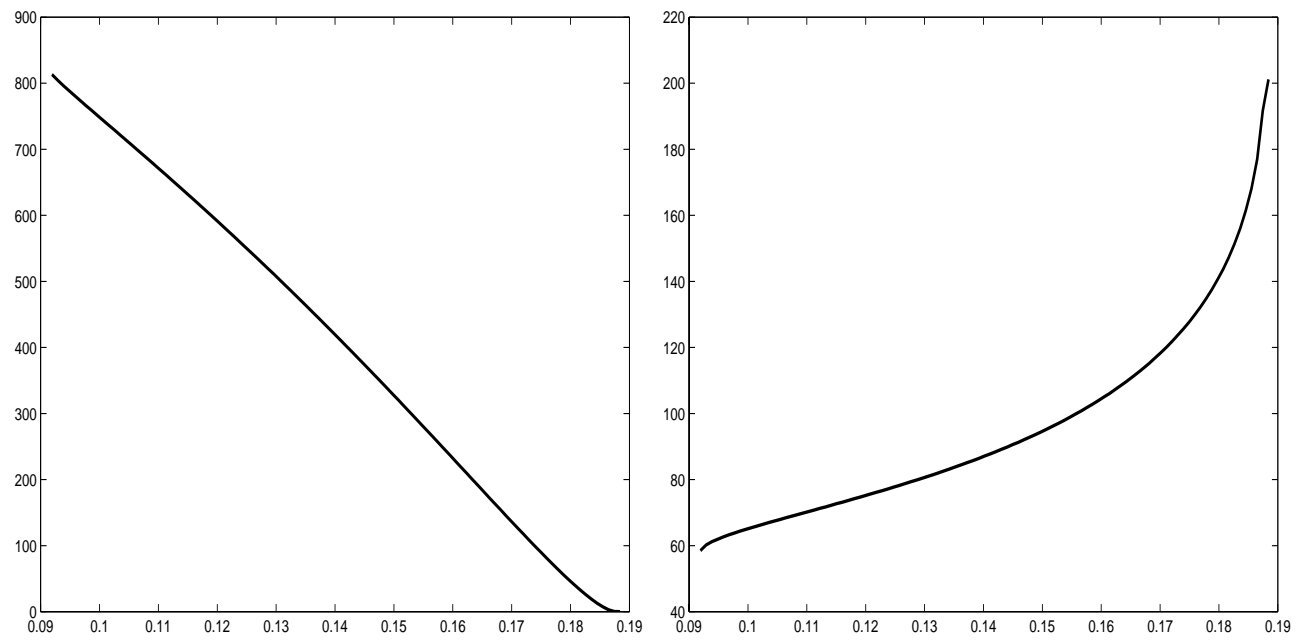

FIG. 9. Moment of instability (left) and minimum (right) of solitons with the endstate equal to the vapor saddle point of Figures 3 and $4\left(v_{\infty}=201.179\right)$.
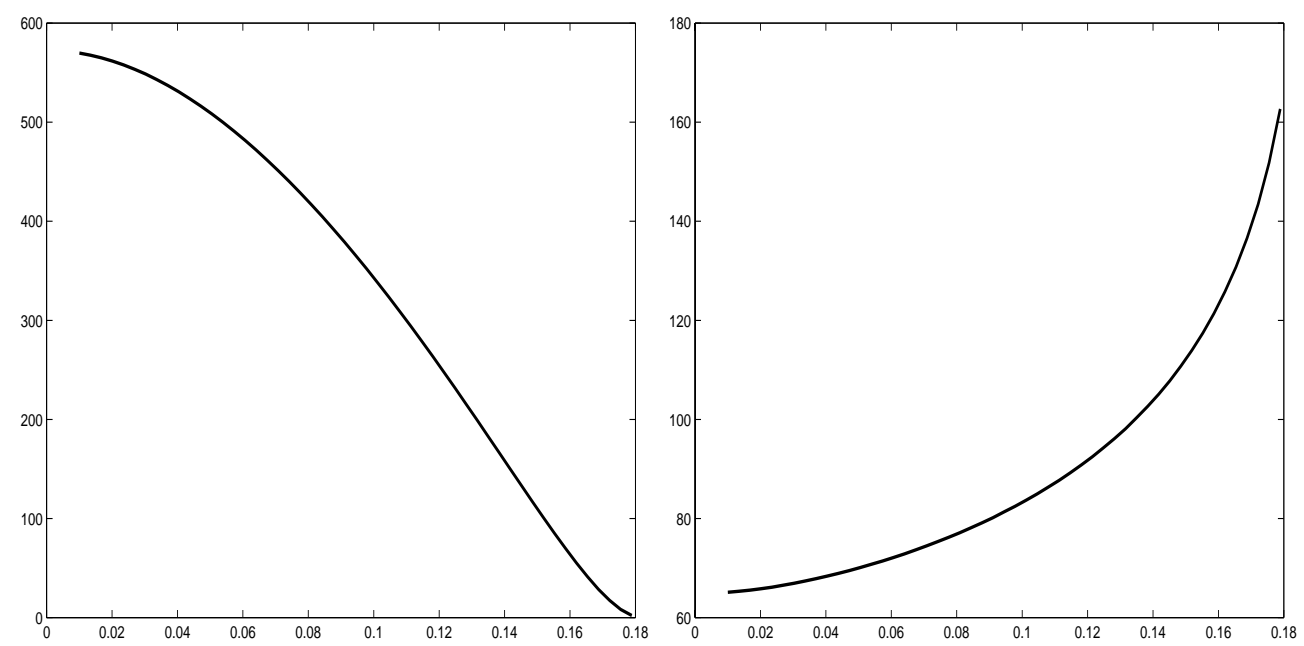

FIG. 10. Moment of instability (left) and minimum (right) of solitons with the endstate equal to a vapor metastable point $\left(v_{\infty}=180\right)$.

Computations with really metastable states instead of the limiting Maxwell point have given similar results. This suggests that the temperate mixed liquid solitons of low "speed" (in fact, physically of low mass transfer) are unstable. A similar result is known for solitons in the Boussinesq water waves theory [9].

REMARK 11 Figure 9 concerns temperate mixed vapor solitons, where the endstate is in the stable vapor phase. They exist again for $\tau^{2}$ in some finite interval $\left(S_{m}, S_{M}\right)$, and when $\tau^{2}$ tends to the minimal value $S_{m}$, the minimum of the soliton tends to the liquid saddle point of the hereteroclinic 

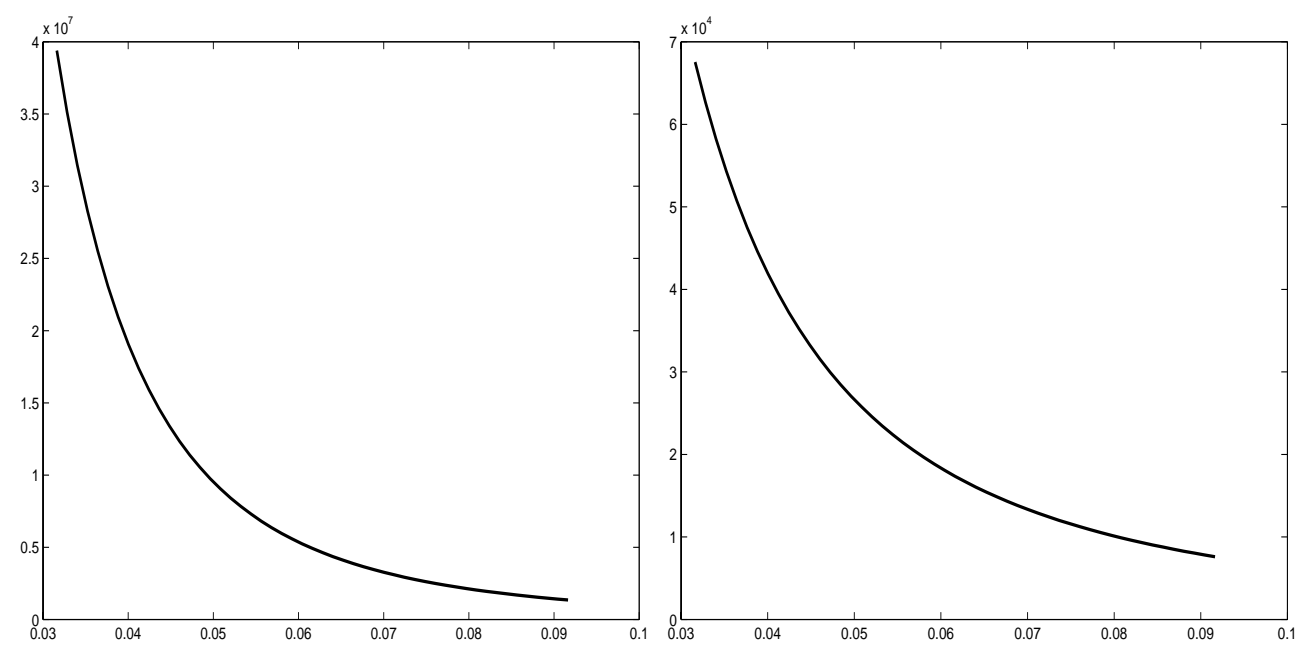

FIG. 11. Moment of instability (left) and maximum (right) of solitons with the endstate equal to the liquid saddle point of Figures 4 and $5\left(v_{\infty}=50.018\right)$.

connection in Figure 4. The graph of the moment of instability looks more complex, being convex for either low or large "speed", but concave in between. The region of concavity seems to correspond to when the minimum of the soliton lies in the metastable or unstable region. However, this rough observation is not confirmed in Figure 10, which concerns also temperate mixed vapor solitons but with a metastable endstate. Indeed, if the graph of $m$ displays a concave part near $\tau=0$, it also has a convex part for large enough $\tau^{2}$, even though the minimum of the soliton is always metastable.

REMARK 12 Figure 11 shows what happens for large mixed liquid solitons. They exist for $\tau^{2}$ in some interval $\left(S_{0}, S_{m}\right)$, the maximum value $S_{m}$ corresponding to a break down of the homoclinic orbit into a set composed of the hereteroclinic connections in Figure 4 and a pure vapor homoclinic connection. The graph of the moment of instability of those rather unusual solitons has shown no failure of convexity, suggesting that they are all stable.

3.3.b Kinks. We now turn to the stability analysis of a kink $\underline{\mathbf{U}}=(\underline{\nu}, \underline{u})$ with endstates $\mathbf{U}_{-}=$ $\left(v_{-}, u_{-}\right), \mathbf{U}_{+}=\left(v_{+}, u_{+}\right)$and speed $\sigma$. We consider the functionals

$$
\begin{aligned}
\mathcal{H}[\mathbf{U} ; \underline{\mathbf{U}}] & :=\int_{-\infty}^{+\infty}(H(\mathbf{U}, \partial \mathbf{U})-H(\underline{\mathbf{U}}, \partial \underline{\mathbf{U}})) \mathrm{d} \xi \\
\mathcal{Q}[\mathbf{U}] & :=\int_{-\infty}^{+\infty}(v u-\underline{v u}) \mathrm{d} \xi \\
\mathcal{P}_{1}[\mathbf{U}] & :=\int_{-\infty}^{+\infty}(v-\underline{v}) \mathrm{d} \xi, \quad \mathcal{P}_{2}[\mathbf{U}]:=\int_{-\infty}^{+\infty}(u-\underline{u}) \mathrm{d} \xi
\end{aligned}
$$

They are well defined on the dense subset of the affine space $\underline{\mathbf{U}}+\left(H^{1} \times L^{2}\right)$ made of functions $\mathbf{U}$ such that $\mathbf{U}-\underline{\mathbf{U}}$ belongs to $L^{1} \times L^{1}$. (Note that this property is useful not only for $\mathcal{P}_{i}$ but also for $\mathcal{H}$ because of the non-linear term in $v$, which is asymptotically linear, unlike what happens 
for solitons.) We call $\lambda_{0}$ the Lagrange multiplier associated with the quadratic functional $\mathcal{Q}$, that is, $\lambda_{0}=\sigma$ in the Eulerian framework or $\lambda_{0}=-\sigma$ in the Lagrangian framework. The other two Lagrange multipliers, $\lambda_{1}, \lambda_{2}$, associated with $\mathcal{P}_{1}$ and $\mathcal{P}_{2}$ respectively, are defined by

$$
\lambda_{i}=\left(\delta \mathcal{H}\left[\mathbf{U}_{ \pm}\right]+\sigma \mathbf{J}_{\varepsilon} \mathbf{U}_{ \pm}\right)_{i}, \quad i=1,2,
$$

the choice of the \pm sign being indifferent due to the jump conditions (necessary for the profile to exist). Then we define a compound functional

$$
\mathcal{F}:=\mathcal{H}-\lambda_{0} \mathcal{Q}-\lambda_{1} \mathcal{P}_{1}-\lambda_{2} \mathcal{P}_{2}
$$

It will be crucial in the following that $\mathcal{F}$ does not change when the profile is translated, which amounts to requiring that

$$
H\left(\mathbf{U}_{+}, 0\right)-\lambda_{0} v_{+} u_{+}-\lambda_{1} v_{+}-\lambda_{2} u_{+}=H\left(\mathbf{U}_{-}, 0\right)-\lambda_{0} v_{-} u_{-}-\lambda_{1} v_{-}-\lambda_{2} u_{-} .
$$

But this is an easy consequence of the equation defining the profile

$$
\left(\delta \mathcal{H}-\lambda_{0} \delta \mathcal{Q}-\lambda_{1} \delta \mathcal{P}_{1}-\lambda_{2} \delta \mathcal{P}_{2}\right)[\underline{\mathbf{U}}] \equiv 0 .
$$

Indeed, taking the inner product of this equation and $\partial \underline{\mathbf{U}}$ and integrating on $\mathbb{R}$ we get the equality above using the fact that

$$
\int \partial \underline{\mathbf{U}} \cdot \delta \mathcal{H}[\underline{\mathbf{U}}]=\int \partial(H(\underline{\mathbf{U}}, \partial \underline{\mathbf{U}}))=H\left(\mathbf{U}_{+}, 0\right)-H\left(\mathbf{U}_{-}, 0\right),
$$

and similarly

$$
\int \partial \underline{\mathbf{U}} \cdot \delta \mathcal{Q}[\underline{\mathbf{U}}]=v_{+} u_{+}-v_{-} u_{-}, \quad \int \partial \underline{\mathbf{U}} \cdot \delta \mathcal{P}_{1}[\underline{\mathbf{U}}]=v_{+}-v_{-}, \quad \int \partial \underline{\mathbf{U}} \cdot \delta \mathcal{P}_{2}[\underline{\mathbf{U}}]=u_{+}-u_{-} .
$$

As a consequence of this invariance property, we have

$$
\mathcal{F}[\widehat{\mathbf{U}}(t)]=\mathcal{F}[\underline{\mathbf{U}}]=0
$$

for all $t$. Therefore, by Lemmas 1 and 2, $\mathcal{F}$ is well defined-the integral being at least semiconvergent - and remains equal to 0 along any solution of 3.15 such that $\mathbf{U}-\underline{\mathbf{U}}$ belongs to $\mathcal{C}\left([0, T] ; H^{3} \times H^{2}\right)$-or equivalently $\mathbf{U}-\widehat{\mathbf{U}} \in \mathcal{C}\left([0, T] ; H^{3} \times H^{2}\right)$-and $\mathbf{U}(0)-\underline{\mathbf{U}}(0)$ belongs to $L^{1} \times L^{1}$.

In the framework of Lemma 3 , we have the following properties.

(P1) The endstates $\mathbf{U}_{ \pm}$are hyperbolic fixed points of the ODE

$$
\left(\delta \mathcal{H}-\lambda_{0} \delta \mathcal{Q}-\lambda_{1} \delta \mathcal{P}_{1}-\lambda_{2} \delta \mathcal{P}_{2}\right)[\mathbf{U}]=0 .
$$

(P2) The set of solutions of the "boundary value problem"

$$
\left\{\begin{array}{l}
\left(\delta \mathcal{H}-\lambda_{0} \delta \mathcal{Q}-\lambda_{1} \delta \mathcal{P}_{1}-\lambda_{2} \delta \mathcal{P}_{2}\right)[\mathbf{U}]=0 \\
\mathbf{U}( \pm \infty)=\mathbf{U}_{ \pm}
\end{array}\right.
$$

is the one-dimensional manifold $\mathbb{M}:=\left\{\mathbf{U}=\underline{\mathbf{U}}_{s} ; s \in \mathbb{R}\right\}$ where $\underline{\mathbf{U}}$ is a fixed profile and $\underline{\mathbf{U}}_{s}(\xi)=\underline{\mathbf{U}}(\xi+s)$. 
(P3) The functional

$$
\mathcal{F}[\mathbf{U}]=\int\left(H(\mathbf{U}, \partial \mathbf{U})-H(\underline{\mathbf{U}}, \partial \underline{\mathbf{U}})-\lambda_{0}(v u-\underline{v} \underline{u})-\lambda_{1}(v-\underline{v})-\lambda_{2}(u-\underline{u})\right)
$$

is independent of $t$ for $\mathbf{U}=\mathbf{U}(t)$ any (classical) solution of 3.15 such that $\mathbf{U}-\underline{\mathbf{U}}$ belongs to $\mathcal{C}\left([0, T] ; H^{3} \times H^{2}\right)$ and $\mathbf{U}(0)-\underline{\mathbf{U}}(0)$ belongs to $L^{1} \times L^{1}$. Furthermore, it equals 0 along the manifold $\mathbb{M}$, as does its variational gradient.

(P4) The Hessian of $\mathcal{F}$ is a monotone operator at all points of $\mathbb{M}$. Furthermore, there exists $c>0$ such that for all $s \in \mathbb{R}$ and $\dot{\mathbf{U}} \in \mathcal{D}(\mathbb{R})$ with $\int \dot{v} \partial \underline{v}_{s}=0$,

$$
\int \dot{\mathbf{U}} \operatorname{Hess} \mathcal{F}\left[\underline{\mathbf{U}}_{s}\right] \cdot \dot{\mathbf{U}} \geqslant c\left(\|\dot{v}\|_{H^{1}(\mathbb{R})}^{2}+\|\dot{u}\|_{L^{2}(\mathbb{R})}^{2}\right) .
$$

(The fact that $c$ is independent of $s$ trivially follows from the fact that $H^{k}$ norms are invariant under translation.)

Assuming $(\mathbf{P 1})(\mathbf{P 4})$, we now investigate the orbital stability of the traveling wave solution $(x, t) \mapsto \widehat{\mathbf{U}}(x, t)=\underline{\mathbf{U}}(x-\sigma t)$ of $(3.15)$.

Our method is very much inspired from the solitary waves stability analysis. In particular, we first show a lemma that will enable us to "factor out" the translation along $\mathbb{M}$. It is analogous to Lemma 3.2 p. 169 in [18] (or Lemma 4.1 p. 405 in [10]), the main difference being that we have to work in an affine space $\underline{\mathbf{U}}+H^{k}(\mathbb{R})$ instead of merely $H^{k}(\mathbb{R})$.

LEMMA 5 For any non-constant function $\underline{U}$ tending exponentially fast to $U_{ \pm}$at $\pm \infty$, for $k \geqslant 0$, there exists $\varepsilon>0$ and a smooth function

$$
\mathcal{S}_{k}: \mathbb{U}_{\varepsilon}=\left\{U \in \underline{U}+H^{k}(\mathbb{R}) ; \inf _{s \in \mathbb{R}}\left\|U-\underline{U}_{s}\right\|_{H^{k}(\mathbb{R})}<\varepsilon\right\} \rightarrow \mathbb{R}
$$

such that

- $\int\left(U_{\mathcal{S}_{k}(U)}-\underline{U}\right) \partial \underline{U}=0$ for all $U \in \mathbb{U}_{\varepsilon}$,

- $\mathcal{S}_{k}\left(U_{r}\right)=\mathcal{S}_{k}(U)-r$ for all $r \in \mathbb{R}$.

Proof. We recall that the notation $U_{s}$ for any function $U$ stands for the function translated by $s$, that is, $U_{s}(\xi)=U(\xi+s)$. Applying the implicit function theorem to the mapping

$$
(s, U) \mapsto \int\left(U_{s}-\underline{U}\right) \partial \underline{U}
$$

about $(s, U)=(0, \underline{U})$, we find $\varepsilon>0$, a neighborhood $\mathcal{W}_{\varepsilon}$ of 0 in $\mathbb{R}$, a neighborhood $\mathbb{V}_{\varepsilon}$ of 0 in $H^{k}(\mathbb{R})$ and a function $\mathcal{S}_{k}$ defined on $\mathbb{V}_{\varepsilon}$ such that

$$
\left(\int\left(U_{s}-\underline{U}\right) \partial \underline{U}=0 \text { and } s \in \mathcal{W}_{\varepsilon}, U \in \underline{U}+\mathbb{V}_{\varepsilon}\right) \Leftrightarrow s=\mathcal{S}_{k}(U) .
$$

Now, by the invariance of the $H^{k}$ norm under translation, $U \in \underline{U}_{t}+\mathbb{V}_{\varepsilon}$ is equivalent to $U_{-t} \in$ $\underline{U}+\mathbb{V}_{\varepsilon}$. Consequently, we have

$$
\left(\int\left(U_{s-t}-\underline{U}\right) \partial \underline{U}=0 \text { and } s \in \mathcal{W}_{\varepsilon}, U_{-t} \in \underline{U}+\mathbb{V}_{\varepsilon}\right) \Leftrightarrow s=\mathcal{S}_{k}\left(U_{-t}\right) .
$$


So we may define

$$
\mathcal{S}_{k}(U)=\mathcal{S}_{k}\left(U_{-t}\right)-t
$$

with values in $-t+\mathcal{W}_{\varepsilon}$. This extends smoothly $\mathcal{S}_{k}$ to

$$
\mathbb{U}_{\varepsilon}=\left\{U \in \underline{U}+H^{k}(\mathbb{R}) ; \inf _{s \in \mathbb{R}}\left\|U-\underline{U}_{s}\right\|_{H^{k}(\mathbb{R})}<\varepsilon\right\},
$$

as claimed_provided that the ball of center 0 and radius $\varepsilon$ is included in $\mathbb{V}_{\varepsilon}$. Moreover, the set $\mathbb{U}_{\varepsilon}$ is by construction invariant under translation, and for all $U \in \mathbb{U}_{\varepsilon}$ and $r \in \mathbb{R}$,

$$
\mathcal{S}_{k}\left(U_{r}\right)=\mathcal{S}_{k}\left(U_{r-t}\right)-t
$$

if $U_{r} \in \underline{U}_{t}+\mathbb{V}_{\varepsilon}$, which is equivalent to $U \in \underline{U}_{t-r}+\mathbb{V}_{\varepsilon}$ and thus implies

$$
\mathcal{S}_{k}(U)=\mathcal{S}_{k}\left(U_{r-t}\right)-(t-r) .
$$

Taking the difference between these two equalities, we get the expected formula

$$
\mathcal{S}_{k}\left(U_{r}\right)=\mathcal{S}_{k}(U)-r .
$$

The next step is to expand the functional $\mathcal{F}$. Brutal Taylor expansion is not valid a priori because $\mathcal{F}$ is not defined on the whole space $\mathbf{U} \in \underline{\mathbf{U}}+\left(H^{2}(\mathbb{R}) \times L^{2}(\mathbb{R})\right)$. However, we may expand each of the integrands separately. In this way, we find that for any $\mathbf{U} \in \underline{\mathbf{U}}+\left(H^{2}(\mathbb{R}) \times L^{2}(\mathbb{R})\right)$, as soon as the integral in $\mathcal{F}[\mathbf{U}]$ is convergent,

$$
\begin{aligned}
& \mathcal{F}[\mathbf{U}]=\int\left(\delta \mathcal{F}[\underline{\mathbf{U}}] \cdot(\mathbf{U}-\underline{\mathbf{U}})+\frac{1}{2}(\mathbf{U}-\underline{\mathbf{U}})^{\mathrm{t}} \operatorname{Hess} \mathcal{F}[\underline{\mathbf{U}}](\mathbf{U}-\underline{\mathbf{U}})\right. \\
&\left.+\alpha(v, \underline{v})(v-\underline{v})^{3}+\beta(\nu, \underline{v})(v-\underline{v})^{2} \partial(\nu-\underline{v})+\gamma(\nu, \underline{v})(v-\underline{v})(\partial v-\partial \underline{v})^{2}\right)
\end{aligned}
$$

where the $L^{\infty}$ norms of the coefficients $\alpha, \beta$ and $\gamma$ depend continuously on $\|v-\underline{v}\|_{L^{\infty}(\mathbb{R})}$, and on $\|\underline{v}\|_{L^{\infty}(\mathbb{R})}$, which is fixed. Since $\mathcal{F}\left[\underline{\mathbf{U}}_{S}\right]=0$ the same expansion holds true with $\underline{\mathbf{U}}$ replaced by $\underline{\mathbf{U}}_{s}$ for any $s$. And by $(\mathbf{P 2}), \delta \mathcal{F}\left[\underline{\mathbf{U}}_{s}\right]=0$, so the first term is null. Therefore, by (P4) the Sobolev embedding $H^{1} \hookrightarrow L^{\infty}$ and Lemma 5 , for $M>0$ small enough, there exists $C_{M}$ so that

$$
\mathcal{F}[\mathbf{U}] \geqslant c\left(\|\tilde{v}-\underline{v}\|_{H^{1}(\mathbb{R})}^{2}+\|\tilde{u}-\underline{u}\|_{L^{2}(\mathbb{R})}^{2}\right)-C_{M}\|\tilde{v}-\underline{v}\|_{H^{1}(\mathbb{R})}^{3}
$$

if

$$
\|\widetilde{v}-\underline{v}\|_{L^{\infty}(\mathbb{R})} \leqslant M, \quad \text { with } \tilde{\mathbf{U}}=\left(\begin{array}{c}
\tilde{v} \\
\tilde{u}
\end{array}\right):=\mathbf{U}_{\mathcal{S}_{1}(v)}
$$

In particular, if

$$
\|\widetilde{v}-\underline{v}\|_{H^{1}(\mathbb{R})} \leqslant \min \left(c /\left(2 C_{M}\right), M\right)
$$

then

$$
\mathcal{F}[\mathbf{U}] \geqslant \frac{c}{2}\left(\|\widetilde{v}-\underline{v}\|_{H^{1}(\mathbb{R})}^{2}+\|\tilde{u}-\underline{u}\|_{L^{2}(\mathbb{R})}^{2}\right) .
$$

Theorem 3 Assume that (P1) (P) hold true. There exists $\varepsilon_{1}>0$ such that for all $\varepsilon \in\left(0, \varepsilon_{1}\right]$ there exists $\eta>0$ so that if $\mathbf{U} \in \underline{\mathbf{U}}+\mathcal{C}\left([0, T) ; H^{3} \times H^{2}\right)$ is a solution of 3.15) with

$$
\max \left(\|\mathbf{U}(0)-\underline{\mathbf{U}}\|_{H^{1} \times L^{2}},\|\mathbf{U}(0)-\underline{\mathbf{U}}\|_{L^{1} \times L^{1}}\right)<\eta
$$

then

$$
\inf _{s \in \mathbb{R}}\left\|\mathbf{U}(t)-\underline{\mathbf{U}}_{S}\right\|_{H^{1} \times L^{2}}<\varepsilon
$$

for all $t \in[0, T)$. 
Proof. By (P3) and the inequality obtained just before the statement of the theorem, we have

$$
\mathcal{F}[\mathbf{U}(0)]=\mathcal{F}[\mathbf{U}(t)] \geqslant \frac{c}{2}\left(\|\widetilde{v}(t)-\underline{v}\|_{H^{1}(\mathbb{R})}^{2}+\|\widetilde{u}(t)-\underline{u}\|_{L^{2}(\mathbb{R})}^{2}\right)
$$

as long as

$$
\|\widetilde{v}(t)-\underline{v}\|_{H^{1}(\mathbb{R})}<\varepsilon_{1}:=\min \left(c /\left(2 C_{1}\right), 1\right) .
$$

Note that by continuity of the map

$$
v \in \underline{v}+\mathbb{V}_{\varepsilon} \mapsto \widetilde{v}=v_{\mathcal{S}_{1}(v)} \in \underline{v}+H^{1}(\mathbb{R}),
$$

we have initially

$$
\|\widetilde{v}(0)-\underline{v}\|_{H^{1}(\mathbb{R})}<\varepsilon 1
$$

provided that

$$
\|v(0)-\underline{v}\|_{H^{1}(\mathbb{R})} \leqslant \eta_{0}
$$

with $\eta_{0}$ (less than the $\varepsilon$ of Lemma 5) small enough.

Take $\varepsilon_{2} \in\left(0, \varepsilon_{1}\right]$. Since $\mathcal{F}$ is continuous on $\left(H^{1} \times L^{2}\right) \cap\left(L^{1} \times L^{1}\right)$, there exists $\eta \in\left(0, \eta_{0}\right]$ such that

$$
\max \left(\|\mathbf{U}(0)-\underline{\mathbf{U}}\|_{H^{1} \times L^{2}},\|\mathbf{U}(0)-\underline{\mathbf{U}}\|_{L^{1} \times L^{1}}\right)<\eta
$$

implies

$$
\mathcal{F}[\mathbf{U}(0)]<\frac{c}{2} \varepsilon_{2}^{2}
$$

Therefore, for all $t \in[0, T)$,

$$
\|\widetilde{\mathbf{U}}(t)-\underline{\mathbf{U}}\|_{H^{1} \times L^{2}}<\varepsilon_{2} .
$$

Recalling that $\widetilde{\mathbf{U}}(t)$ is a translate of $\mathbf{U}(t)$, this completes the proof.

\section{Appendix A. Selection of Hamiltonian operators by Olver's method}

We first consider a one-dimensional differential operator $\mathcal{A}$, acting on functionals of dependent variables $(v, u, w)$ and taking the form

$$
\mathcal{A}=\left(\begin{array}{ccc}
0 & a(\mathbf{u}) \mathrm{D}_{x} & 0 \\
\mathrm{D}_{x} a(\mathbf{u}) & 0 & \mathrm{D}_{x} b(\mathbf{u}) \mathrm{D}_{x} \\
0 & -\mathrm{D}_{x} b(\mathbf{u}) \mathrm{D}_{x} & 0
\end{array}\right),
$$

where $\mathrm{D}_{x}$ stands for the total derivative with respect to the single independent variable $x$, and $a$ and $b$ are arbitrary scalar functions of $\mathbf{u}=(v, u, w)$. The operator $\mathcal{A}$ is obviously skewsymmetric for the $L^{2}$ inner product.

Recall from [30, p. 444] the following

Theorem 4 The bracket $\{\cdot, \cdot\}$ acting on functionals $\mathcal{P}: \mathbf{u} \mapsto \int P[\mathbf{u}] \mathrm{d} x, \mathcal{Q}: \mathbf{u} \mapsto \int Q[\mathbf{u}] \mathrm{d} x$ as

$$
\{\mathcal{P}, \mathcal{Q}\}: \mathbf{u} \mapsto \int \delta \mathcal{P}[\mathbf{u}] \cdot \mathcal{A} \delta \mathcal{Q}[\mathbf{u}] \mathrm{d} x
$$


satisfies Jacobi's identity if and only if

$$
\int \operatorname{pr} \mathbf{v}_{\mathcal{A} \boldsymbol{\theta}}(\boldsymbol{\theta} \wedge \mathcal{A} \boldsymbol{\theta}) \mathrm{d} x=0,
$$

where $\boldsymbol{\theta}=(\eta, \theta, \zeta), \eta=\mathrm{d} v, \theta=\mathrm{d} u, \zeta=\mathrm{d} w$ being "canonical" 1-forms, $\mathcal{A} \boldsymbol{\theta}$ has three components $(\mathcal{A} \boldsymbol{\theta})^{1},(\mathcal{A} \boldsymbol{\theta})^{2},(\mathcal{A} \boldsymbol{\theta})^{3}$ defined by letting $\mathcal{A}$ act on $\boldsymbol{\theta}$ through the formulae

$$
\mathrm{D}_{x} \eta=\eta_{x}:=\mathrm{d} v_{x}, \quad \mathrm{D}_{x} \theta=\theta_{x}:=\mathrm{d} u_{x}, \quad \mathrm{D}_{x} \zeta=\zeta_{x}:=\mathrm{d} w_{x}, \quad \text { etc. }
$$

the wedge product $\boldsymbol{\theta} \wedge \mathcal{A} \boldsymbol{\theta}$ stands for the extended 2-form

$$
\boldsymbol{\theta} \wedge \mathcal{A} \boldsymbol{\theta}=\eta \wedge(\mathcal{A} \boldsymbol{\theta})^{1}+\theta \wedge(\mathcal{A} \boldsymbol{\theta})^{2}+\zeta \wedge(\mathcal{A} \boldsymbol{\theta})^{3},
$$

and $\mathbf{v}_{\mathcal{A} \theta}$ is the vector field

$$
\mathbf{v}_{\mathcal{A} \boldsymbol{\theta}}=(\mathcal{A} \boldsymbol{\theta})^{1} \frac{\partial}{\partial v}+(\mathcal{A} \boldsymbol{\theta})^{2} \frac{\partial}{\partial u}+(\mathcal{A} \boldsymbol{\theta})^{3} \frac{\partial}{\partial w},
$$

prolongated to

$$
\operatorname{pr} \mathbf{v}_{\mathcal{A} \boldsymbol{\theta}}=\mathbf{v}_{\mathcal{A} \boldsymbol{\theta}}+\mathrm{D}_{x}\left((\mathcal{A} \boldsymbol{\theta})^{1}\right) \frac{\partial}{\partial v_{x}}+\mathrm{D}_{x}\left((\mathcal{A} \boldsymbol{\theta})^{2}\right) \frac{\partial}{\partial u_{x}}+\mathrm{D}_{x}\left((\mathcal{A} \boldsymbol{\theta})^{3}\right) \frac{\partial}{\partial w_{x}}+\cdots,
$$

which acts on extended 2-forms as an exterior differentiation.

Using this theorem, it is rather easy to discriminate among skewsymmetric operators those which satisfy Jacobi's identity. In fact, it can be cumbersome to write down $\int \operatorname{pr} \mathbf{v}_{\mathcal{A}} \boldsymbol{\theta}(\boldsymbol{\theta} \wedge \mathcal{A} \boldsymbol{\theta}) \mathrm{d} x$. Instead, as suggested by Olver, one may observe that

$$
\int \operatorname{pr} \mathbf{v}_{\mathcal{A}} \boldsymbol{\theta}(\boldsymbol{\theta} \wedge \mathcal{A} \boldsymbol{\theta}) \mathrm{d} x=2 \operatorname{pr} \mathbf{v}_{\mathcal{A} \boldsymbol{\theta}} \Theta, \quad \Theta:=\frac{1}{2} \int(\boldsymbol{\theta} \wedge \mathcal{A} \boldsymbol{\theta}) \mathrm{d} x,
$$

whose expression can be greatly simplified through integrations by parts.

In our case, we have

$$
\begin{gathered}
(\mathcal{A} \boldsymbol{\theta})^{1}=a(\mathbf{u}) \theta_{x}, \quad(\mathcal{A} \boldsymbol{\theta})^{2}=\mathrm{D}_{x}(a(\mathbf{u}) \eta)+\mathrm{D}_{x}\left(b(\mathbf{u}) \zeta_{x}\right), \quad(\mathcal{A} \boldsymbol{\theta})^{3}=-\mathrm{D}_{x}\left(b(\mathbf{u}) \theta_{x}\right), \\
\mathbf{v}_{\mathcal{A} \boldsymbol{\theta}}=\left(a(\mathbf{u}) \theta_{x}\right) \frac{\partial}{\partial v}+\mathrm{D}_{x}\left(a(\mathbf{u}) \eta+b(\mathbf{u}) \zeta_{x}\right) \frac{\partial}{\partial u}-\mathrm{D}_{x}\left(b(\mathbf{u}) \theta_{x}\right) \frac{\partial}{\partial w},
\end{gathered}
$$

and $\Theta$ reduces to

$$
\Theta=\int\left(a(\mathbf{u}) \eta \wedge \theta_{x}+b(\mathbf{u}) \zeta_{x} \wedge \theta_{x}\right) \mathrm{d} x
$$

Therefore,

$$
\operatorname{pr} \mathbf{v}_{\mathcal{A}} \boldsymbol{\theta} \Theta=\mathbf{v}_{\mathcal{A}} \boldsymbol{\theta} \Theta,
$$

where there are still many terms if $a$ and $b$ do depend on the three variables. However, if $a$ and $b$ depend only on the first dependent variable $v$, we find that

$$
\left.\operatorname{pr} \mathbf{v}_{\mathcal{A} \theta} \Theta=\int a(v) a^{\prime}(v) \theta_{x} \wedge \eta \wedge \theta_{x}+a(v) b^{\prime}(v) \theta_{x} \wedge \zeta_{x} \wedge \theta_{x}\right) \mathrm{d} x=0
$$


because of $\theta_{x}$ appearing twice in the wedge products. This applies in particular to the operator $\mathcal{A}$ of Section 1.3

We now consider the multidimensional operator of Section 1.1 (save for the minus sign)

$$
\mathcal{J}=\left(\begin{array}{ccc}
0 & \operatorname{div} & 0 \\
\nabla & 0 & s \nabla \\
0 & \operatorname{div} s \cdot & 0
\end{array}\right)
$$

with dependent variables $(\rho, \mathbf{u}, S)$, and $s=S / \rho$. Of course there is a multidimensional version of Theorem 4, which is actually the one given in [30]. To apply it, we need notations. We denote here by $u^{1}, u^{2}, u^{3}$ the components of the velocity field $\mathbf{u}$, and $\eta=\mathrm{d} \rho, \theta^{i}=\mathrm{d} u^{i}, \zeta=\mathrm{d} S$,

$$
\mathrm{D}_{x_{j}} \eta=\eta_{j}:=\mathrm{d} v_{x_{j}}, \quad \mathrm{D}_{x_{j}} \theta^{i}=\theta_{j}^{i}:=\mathrm{d} u_{x_{j}}^{i}, \quad \mathrm{D}_{x_{j}} \zeta=\zeta_{x_{j}}:=\mathrm{d} S_{x_{j}}, \quad \text { etc. }
$$

And here $\boldsymbol{\theta}$ stands for $\left(\eta, \theta^{1}, \theta^{2}, \theta^{3}, \zeta\right)$. We have

$$
\mathbf{v}_{\mathcal{J} \boldsymbol{\theta}}=\left(\theta_{1}^{1}+\theta_{2}^{2}+\theta_{3}^{3}\right) \frac{\partial}{\partial \rho}+\sum_{j=1}^{3}\left(\eta_{j}+s \zeta_{j}\right) \frac{\partial}{\partial u^{j}}+\left(\mathrm{D}_{x_{1}}\left(s \theta^{1}\right)+\mathrm{D}_{x_{2}}\left(s \theta^{2}+\mathrm{D}_{x_{3}}\left(s \theta^{3}\right)\right) \frac{\partial}{\partial S}\right.
$$

and

$$
\boldsymbol{\theta} \wedge \mathcal{J} \boldsymbol{\theta}=\eta \wedge\left(\theta_{1}^{1}+\theta_{2}^{2}+\theta_{3}^{3}\right)+\sum_{j=1}^{3} \theta^{j} \wedge\left(\eta_{j}+s \zeta_{j}\right)+\zeta \wedge\left(\mathrm{D}_{x_{1}}\left(s \theta^{1}\right)+\mathrm{D}_{x_{2}}\left(s \theta^{2}+\mathrm{D}_{x_{3}}\left(s \theta^{3}\right)\right) .\right.
$$

Therefore, $\Theta$ reduces to

$$
\Theta=\int s\left(\theta^{1} \wedge \zeta_{1}+\theta^{2} \wedge \zeta_{2}+\theta^{3} \wedge \zeta_{3}\right) \mathrm{d} x,
$$

and

$$
\begin{aligned}
\operatorname{pr} \mathbf{v}_{\mathcal{J} \theta} \Theta=\int\left(\left(\frac{\partial s}{\partial \rho}\right)\left(\theta_{1}^{1}+\theta_{2}^{2}+\theta_{3}^{3}\right)+\left(\frac{\partial s}{\partial S}\right)\left(\mathrm{D}_{x_{1}}\left(s \theta^{1}\right)+\right.\right. & \left.\left.\mathrm{D}_{x_{2}}\left(s \theta^{2}+\mathrm{D}_{x_{3}}\left(s \theta^{3}\right)\right)\right)\right) \\
& \wedge\left(\theta^{1} \wedge \zeta_{1}+\theta^{2} \wedge \zeta_{2}+\theta^{3} \wedge \zeta_{3}\right) \mathrm{d} x .
\end{aligned}
$$

Since

$$
\frac{\partial s}{\partial \rho}+s \frac{\partial s}{\partial S}=0, \quad \frac{\partial s}{\partial S}=v
$$

the only remaining terms in $\operatorname{pr} \mathbf{v}_{\mathcal{J}}{ }_{\boldsymbol{\theta}} \Theta$ are of the form

$$
\int v\left(\theta^{i} \wedge \theta^{j} \wedge\left(s_{x_{i}} \zeta_{j}-s_{x_{j}} \zeta_{i}\right)\right) \mathrm{d} x .
$$

So $\operatorname{pr} \mathbf{v}_{\mathcal{J}} \Theta \Theta$ is zero in dimension one only. 
Appendix B. Application of the Sturm-Liouville theory to $\mathcal{L}$

We do the computation in the Lagrangian framework, in which

$$
\mathcal{L}=\left(\begin{array}{cc}
\frac{\mathcal{M}}{\sigma} & \sigma \\
\sigma & 1
\end{array}\right)
$$

and the Sturm-Liouville operator $\mathcal{M}=\underline{\mathcal{M}}-\sigma^{2}$ is known to have the following properties: 1) since the endstate of the soliton $\underline{\mathbf{U}}$ is subsonic the essential spectrum of $\mathcal{M}$ is positive and bounded away from zero; 2) the first derivative of the profile $\underline{v}$, which has only one zero, is in the kernel of $\mathcal{M}$. From the Sturm-Liouville theory [32], this implies that $\mathcal{M}$ admits exactly one negative eigenvalue $\mu_{0}$ and that the eigenvalues different from $\mu_{0}$ and 0 are positive. Furthermore, the eigenspace associated with $\mu_{0}$ is spanned by a positive eigenfunction. Assume now that $\lambda$ is a negative eigenvalue of $\mathcal{L}$, with eigenvector $(\omega, w)^{t}$, that is,

$$
\left(\begin{array}{cc}
\mathcal{M} & \sigma \\
\sigma & 1
\end{array}\right)\left(\begin{array}{l}
\omega \\
w
\end{array}\right)=\lambda\left(\begin{array}{l}
\omega \\
w
\end{array}\right)
$$

Eliminating $w$ we obtain

$$
\mathcal{M} \cdot \omega=\frac{\lambda\left(\lambda-1-\sigma^{2}\right)}{\lambda-1} \omega,
$$

which means that the negative number $\lambda\left(\lambda-1-\sigma^{2}\right) /(\lambda-1)$ must be an eigenvalue of $\mathcal{M}$. Hence

$$
\frac{\lambda\left(\lambda-1-\sigma^{2}\right)}{\lambda-1}=\mu_{0}
$$

Conversely, there exists a unique negative number $\lambda$ satisfying the equality above, since the second order polynomial

$$
\lambda^{2}-\lambda\left(1+\sigma^{2}+\mu_{0}\right)+\mu_{0}
$$

has a unique negative root.

REMARK 13 From the dimensional point of view, this computation looks incorrect. In fact, we can restore the physical homogeneity of equations by inserting a scaling factor (homogeneous to $\sigma^{2}$ ) in the first row of the right-hand side in $(\mathrm{B} .28)$. The vector solution $\mathbf{W}$ obtained this way will not exactly be an eigenvector of $\mathcal{L}$, but it will satisfy

$$
\int \mathbf{W}^{*} \mathcal{L} \mathbf{W}<0
$$

which is precisely what we need in the proof of Proposition 4

\section{REFERENCES}

1. Abeyaratne, R. \& Knowles, J. K. Kinetic relations and the propagation of phase boundaries in solids. Arch. Rat. Mech. Anal. 114 (1991), 119-154. Zbl 0745.73001 MR 1094433

2. Anderson, D. M., McFadden, G. B., \& Wheeler, A. A. Diffuse-interface methods in fluid mechanics. Annual Review of Fluid Mechanics, Vol. 30, Annual Reviews, Palo Alto, CA (1998), 139-165. MR 1609626 
3. Arnold, V. I. \& Khesin, B. A. Topological Methods in Hydrodynamics. Appl. Math. Sci. 125, Springer, New York (1998). Zbl 0902.76001 MR 1612569

4. Benjamin, T. B. Impulse, flow force and variational principles. IMA J. Appl. Math. 32 (1984), 3-68. Zbl 0584.76001 MR 0740456

5. Benzoni-Gavage, S. Linear stability of propagating phase boundaries in capillary fluids. Phys. D $\mathbf{1 5 5}$ (2001), 235-273.

6. Benzoni-Gavage, S., Danchin, R., \& Descombes, S. Well-posedness of multi-dimensional Korteweg models. In preparation (2004).

7. Benzoni-Gavage, S., Danchin, R., \& Descombes, S. Well-posedness of one-dimensional Korteweg models. Preprint (2004).

8. Bethuel, F., Orlandi, G., \& Smets, D. Vortex rings for the Gross-Pitaevskii equation. J. Eur. Math. Soc. 6 (2004), 17-94. Zbl pre02075133 MR 2041006

9. BONA, J. L. \& SACHS, R. L. Global existence of smooth solutions and stability of solitary waves for a generalized Boussinesq equation. Comm. Math. Phys. 118 (1988), 15-29. Zbl 0654.35018 MR 0954673

10. Bona, J. L., Souganidis, P. E., \& Strauss, W. A. Stability and instability of solitary waves of Korteweg-de Vries type. Proc. Roy. Soc. London Ser. A 411 (1987), 395-412. Zbl 0648.76005 MR 0897729

11. Boussinesq, J. Théorie des ondes et des remous qui se propagent le long d'un canal rectangulaire horizontal, en communiquant au liquide contenu dans ce canal des vitesses sensiblement pareilles de la surface au fond. J. Math. Pures Appl. 17 (1872), 55-109. JFM 04.0493.04

12. Bresch, D., Desjardins, B., \& Lin, C.-K. On some compressible fluid models: Korteweg, lubrication, and shallow water systems. Comm. Partial Differential Equations 28 (2003), 843-868. Zbl pre01912722 MR 1978317

13. Chalons, C., Coquel, F., Dehais, G., Jamet, D., \& Lebaigue, O. Extended formulations for van der Waals models. Application to finite volume methods. In preparation.

14. Constantin, A. \& Strauss, W. A. Stability of peakons. Comm. Pure Appl. Math. 53 (2000), 603-610. Zbl 1049.35149 MR 1737505

15. DANChin, R. \& DESJARDIns, B. Existence of solutions for compressible fluid models of Korteweg type. Ann. Inst. H. Poincaré Anal. Non Linéaire 18 (2001), 97-133. Zbl 1010.76075 MR 1810272

16. Dunn, J. E. \& SERrin, J. On the thermomechanics of interstitial working. Arch. Rat. Mech. Anal. 88 (1985), 95-133. Zbl 0582.73004 MR 0775366

17. GaVrilyuk, S. \& Gouin, H. Symmetric form of governing equations for capillary fluids. Trends in Applications of Mathematics to Mechanics (Nice, 1998), Chapman \& Hall/CRC Monogr. Surv. Pure Appl. Math. 106, Chapman \& Hall/CRC, Boca Raton, FL (2000), 306-311. Zbl 0973.35152 MR 1734893

18. Grillakis, M., Shatah, J., \& Strauss, W. Stability theory of solitary waves in the presence of symmetry. I. J. Funct. Anal. 74 (1987), 160-197. Zbl 0656.35122 MR 0901236

19. Hattori, H. \& Li, D. Global solutions of a high-dimensional system for Korteweg materials. J. Math. Anal. Appl. 198 (1996), 84-97. Zbl 0858.35124 MR 1373528

20. Howard, P. \& Zumbrun, K. Pointwise estimates and stability for dispersive-diffusive shock waves. Arch. Rat. Mech. Anal. 155 (2000), 85-169.

21. Howard, P. \& Zumbrun, K. Stability of undercompressive shock profiles. Arch. Rat. Mech. Anal., to appear.

22. Huang, F., Li, H., Matsumura, A., \& Odanaka, S. Well-posedness and stability of multidimensional quantum hydrodynamics for semiconductors in $\mathbb{R}^{3}$. Preprint (2003).

23. Jamet, D., Lebaigue, O., Coutris, N., \& Delhaye, J. M. The second gradient method for the direct numerical simulation of liquid-vapor flows with phase change. J. Comput. Phys. 169 (2001), 624-651. Zbl 1047.76098 MR 1836527 
24. Jamet, D., Torres, D., \& Brackbill, J. U. On the theory and computation of surface tension: the elimination of parasitic currents through energy conservation in the second-gradient method. J. Comput. Phys. 182 (2002), 262-276. Zbl 1058.76597

25. KATO, T. Quasi-linear equations of evolution, with applications to partial differential equations. Spectral Theory and Differential Equations (Dundee, 1974), dedicated to Konrad Jörgens, Lecture Notes in Math. 448, Springer, Berlin (1975), 25-70. Zbl 0315.35077 MR 0407477

26. Korteweg, D. J. Sur la forme que prennent les équations des mouvements des fluides si l'on tient compte des forces capillaires causées par des variations de densité considérables mais continues et sur la théorie de la capillarité dans l'hypothèse d'une variation continue de la densité. Arch. Néer. Sci. Exactes Sér. II 6 (1901), 1-24. JFM 32.0756.02

27. LeFloch, P. G. Hyperbolic Systems of Conservation Laws. The Theory of Classical and Nonclassical Shock Waves. Lectures in Math. ETH Zürich, Birkhäuser, Basel (2002). Zbl 1019.35001 MR 1927887

28. Li, H. \& MARCATI, P. Existence and asymptotic behavior of multi-dimensional quantum hydrodynamic model for semiconductors. Comm. Math. Phys. 245 (2004), 215-247. Zbl pre02048127 MR 2039696

29. Mascia, C. \& Zumbrun, K. Pointwise Green function bounds for shock profiles of systems with real viscosity. Arch. Rat. Mech. Anal. 169 (2003), 177-263. Zbl 1035.35074 MR 2004135

30. Olver, P. J. Applications of Lie Groups to Differential Equations. 2nd ed., Grad. Texts in Math. 107, Springer, New York (1993). Zbl 0785.58003 MR 1240056

31. Pego, R. L. \& Weinstein, M. I. Eigenvalues, and instabilities of solitary waves. Philos. Trans. Roy. Soc. London Ser. A 340 (1992), 47-94. Zbl 0776.35065 MR 1177566

32. Protter, M. H. \& Weinberger, H. F. Maximum Principles in Differential Equations. Springer, New York (1984). Corrected reprint of the 1967 original. Zbl 0549.35002 MR 0762825

33. Rowlinson, J. S. Translation of J. D. van der Waals' "The thermodynamic theory of capillarity under the hypothesis of a continuous variation of density". J. Statist. Phys. 20 (1979), 197-244. MR 0523642

34. Rowlinson, J. S. \& Widom, B. Molecular Theory of Capillarity. Oxford Univ. Press (1982).

35. ŠNOL', È. Behavior of eigenfunctions and the spectrum of Sturm-Liouville operators. Uspekhi Mat. Nauk (N.S.) 9 (1954), no. 4, 113-132 (in Russian). Zbl 0056.34401 MR 0068077

36. Truesdell, C. \& Noll, W. The Nonlinear Field Theories of Mechanics. 2nd ed., Springer, Berlin (1992). Zbl 0779.73004 MR 1215940

37. Weast, R. C., \& Astle, M. J. (eds.) CRC Handbook of Chemistry and Physics. CRC Press, Boca Raton, FL (1980).

38. ZuMbrun, K. Dynamical stability of phase transitions in the $p$-system with viscosity-capillarity. SIAM J. Appl. Math. 60 (2000), 1913-1924. Zbl 0973.35183 MR 1763309

39. Zumbrun, K. Multidimensional stability of planar viscous shock waves. Advances in the Theory of Shock Waves, Progr. Nonlinear Differential Equations Appl. 47, Birkhäuser Boston, Boston, MA (2001), 307-516. Zbl 0989.35089 MR 1842778

40. Zumbrun, K. \& Howard, P. Pointwise semigroup methods and stability of viscous shock waves. Indiana Univ. Math. J. 47 (1998), 741-871. Zbl 0977.35080 MR 1800804 